\title{
Wind Speed Measurement Using Forward Scattered GPS Signals
}

\author{
James L. Garrison, Member, IEEE, Attila Komjathy, Valery U. Zavorotny, and Stephen J. Katzberg
}

\begin{abstract}
Instrumentation and retrieval algorithms are described which use the forward scattered range-coded signals from the global positioning system (GPS) radio navigation system for the measurement of sea surface roughness. This roughness has long been known to be dependent upon the surface wind speed. Experiments were conducted from aircraft along the TOPEX ground track and over experimental surface truth buoys. These flights used a receiver capable of recording the cross-correlation power in the reflected signal. The shape of this power distribution was then compared against analytical models, which employ a geometric optics approach. Two techniques for matching these functions were studied. The first recognized the most significant information content in the reflected signal is contained in the trailing edge slope of the waveform. The second attempted to match the complete shape of the waveform by approximating it as a series expansion and obtaining the nonlinear least squares estimate. Discussion is also presented on anomalies in the receiver operation and their identification and correction.
\end{abstract}

Index Terms-Bistatic radar, global positioning system (GPS), oceanography, retrievals, scatterometry.

\section{INTRODUCTION}

$\mathbf{I}^{1}$ T HAS long been recognized that the reflection of electromagnetic radiation from the ocean surface contains information about the statistical properties of that surface. These statistics are indirectly related to the near-surface meteorological conditions. This relationship forms the basis of most oceanographic radar remote sensing systems. Most experiments to date, however, have used systems employing a dedicated transmitter and receiver. Furthermore, these systems have predominantly been monostatic, measuring the power of the backscattered radiation. The possibility of using the radio navigation signals from the global positioning system (GPS) as a source of illumination in a forward-scattered radar remote sensing instrument for sea surface roughness was first presented, along with preliminary experimental measurement, in [1]. This measurement technique is unique, not only in the use of a bistatic geometry with existing sources of radio frequency illumination, but also in the use of the correlation properties of the pseudo-random noise (PRN) signal transmitted by GPS as

Manuscript received July 14, 2000; revised May 21, 2001.

J. Garrison is with the School of Aeronautics and Astronautics, Purdue University, West Lafayette, IN 47907 USA (e-mail: jgarriso@ecn.purdue.edu).

A. Komjathy is with the Ionospheric and Atmospheric Remote Sensing Group of the Jet Propulsion Laboratory, Pasadena, CA 91109 USA (e-mail: Attila.Komjathy@jpl.nasa.gov).

V. Zavorotny is with the NOAA Environmental Technology Laboratory, Boulder, CO 80303-3328 USA (e-mail: vzavorotny@etl.noaa.gov).

S. Katzberg is with NASA Langley Research Center, Hampton, VA 23681

USA (e-mail: s.j.katzberg@larc.nasa.gov).

Publisher Item Identifier S 0196-2892(02)01410-9. opposed to requiring a direct measurement of received power. Reflected GPS signals have also been proposed to be used as a bistatic satellite altimeter [2], [3].

The technique in [1] measures the shape of the cross-correlation between a reflected signal and the locally generated PRN code. This paper documents the subsequent research performed to mechanize this instrument concept, involving the construction of a specialized GPS receiver and the inversion of bistatic scattering models to estimate wind speed from correlation measurements. Two campaigns of aircraft flights were conducted to provide a determination of the absolute accuracy of this method in comparison to surface truth data, as well as its precision relative to presently operating satellite instruments.

\section{EXPERIMENT DESCRIPTION}

\section{A. Measurement Technique}

Consider first the reflection of a GPS signal from a perfectly flat surface. The reflected signal will come primarily from a distribution of points within the first few Fresnel zones. However, [4] has shown that this approximation leads directly to the geometric optics limit. Therefore, reflections from a perfectly flat surface can be approximated as a single ray path. Other than a change in polarization from exclusively right hand circular to predominantly left hand circular (which can be accounted for with a proper antenna design), the reflected signal will appear identical to a direct signal delayed by the path length difference to the specular point. Consequently when the cross-correlation between this signal and the locally generated PRN code is computed as a function of the relative delay between the two signals, it would ideally be described by the function

$$
\Lambda(\tau)=\left\{\begin{array}{cc}
1,-\left|\frac{\tau}{\tau_{C H I P}}\right| & -\tau_{C H I P} \leq \tau \leq \tau_{C H I P} \\
0, & \text { otherwise }
\end{array}\right\}
$$

in which $\tau$ is the relative delay (in distance) between two codes, and $\tau_{C H I P}$ is the length of one code "chip" or bit transition in the PRN code sequence ( $293 \mathrm{~m}$ for the C/A code used exclusively in this experimentation). For more details on the fundamentals of GPS receiver technology, the interested reader is referred to several textbook references [5] and [6]. Given the incoherent nature of the reflected signal, it is best to work only with power, not voltage signals, therefore the square of this function $\Lambda^{2}(\tau)$ is used from now on.

When the surface is not perfectly flat, but has some random variation in height, then the incident radiation will be reflected from points having a distribution in path length delays near that 
from the specular point. A distribution in surface heights will result in some small fraction of the reflected signal arriving earlier than that from the specular point delay. This effect will be neglected, as justified later in this paper. The more important effect is the slope of surface facets allowing reflections to occur from points away from the specular point, resulting in longer path length delays. The result of this is that all of the reflected signal power no longer arrives with a single time delay, but rather with a continuous distribution in delay. When the cross-correlation is performed, the result will therefore be an infinite sum of the $\Lambda^{2}$ functions at these delays, scaled with the relative power density. This infinite sum can be expressed as a convolution of the reflected power distribution with the $\Lambda^{2}$ function. A more rigorous derivation is provided in [7]

$$
\left\langle\left|Y^{2}(\tau)\right|\right\rangle=\int p(\eta) \Lambda^{2}(\tau-\eta) d \eta .
$$

The power per unit delay $p(\eta)$ is itself computed by the integral of reflected power over all points with delay $\eta$. (The locus of these points forms an ellipse on a flat reflecting surface). Qualitatively, the reflecting surface roughness increases with increasing surface wind speed. The physical mechanism is that high wind will increase the mean square slope, allowing a higher probability of reflections at longer distances from the specular point.

Analytical and empirical models have been generated for this dependence [8], [9] and [4]. The application of them for the retrieval of wind speed will be developed in this paper.

\section{B. Instrumentation}

A GPS receiver development system [10], based upon the MITEL 2010 front-end and 2021 correlator chips, was used to make the first experimental measurements of ocean-reflected GPS signals for this purpose. Software was written for this receiver board to allow direct open-loop control of the correlator code delay and the down conversion frequency for a set of correlator channels operating on the 2 bit digitized signal from a downward-looking left hand circularly polarized (LHCP) antenna. An automatic gain control (AGC) is used such that the noise-like signal has a constant average power prior to correlation. In other words, the sampled signal has a zero mean and a constant variance. This receiver was capable of tracking direct line of sight satellites through a zenith-oriented right hand circularly polarized (RHCP) antenna and recording the cross-correlation function from the same six satellites viewed as reflected signals using a nadir-oriented LHCP antenna. Two modes of operation for the receiver were defined: serial and parallel.

The serial delay mapping receiver (SDMR), tracks up to six direct satellites and generates a position and time solution from their pseudroanges. This position is used to initialize the code delay and Doppler frequency in the reflected channels. The code delay is set to approximate a specular reflection delay of $2 h \sin \gamma$ within the nearest half code chip ( $h$ is the receiver altitude and $\gamma$ is the satellite elevation angle.) It then records the correlation power from the reflected signal in 32 range bins for each of the same six satellites by sequentially stepping through relative delays and performing a coherent integration of the inphase and quadrature components for $1 \mathrm{~ms}$ at each step. The correlation power is then computed as the sum square of these two components. This power in each range bin is passed through a moving average filter and then saved to disk once per second. The commanded code delay of each reflected channel correlator relative to that for the direct (tracking) channel is also saved.

The Parallel Delay Mapping Receiver (PDMR), illustrated in Fig. 1 continuously records the cross-correlation in ten to 12 range bins at fixed delays all from one or two satellites. The coherent integration time is fixed at $1 \mathrm{~ms}$ on the correlator chip hardware. However, the sum square of the inphase and quadrature components is (incoherently) averaged for $0.1 \mathrm{~s}$ before being saved to disk. Furthermore, samples of the relative code phase between the direct and reflected channels are taken at each $0.1 \mathrm{~s}$ interval. This improves the ability to reference the waveform samples to specular point delays. The PDMR has an improvement in signal to noise ratio of $10 \mathrm{~dB}$ over the SDMR at each sample.

In the PDMR, the timing differences between the direct channel and the parallel reflected channels is measured each $0.1 \mathrm{~s}$ to a precision of $1 / 2048$ chip. This knowledge allows subsequent samples to be better aligned with the true estimate of the specular point during postprocessing, taking out much of the error resulting from aircraft motion.

\section{Data Collection Campaigns}

The first collection of correlation data from reflected GPS signals occurred on four aircraft flights between July and November, 1997. These early experiments compared measured waveform shapes collected under a variety of sea state conditions [as measured on buoys operated by the NOAA National Data Buoy Center (NDBC) buoys] [1].

Two aircraft flight campaigns were subsequently conducted in which this receiver was flown in conjunction with surface truth measurements and the TOPEX satellite altimeter. The purpose of these experiments was to provide comparison data for wind speed retrievals from the reflected GPS signal. The NASA Goddard Space Flight Center C-130 aircraft operating out of the NASA Wallops Flight Facility was flown along the TOPEX-Poseidon ground track (cycles 107, 110 and 111, pass no. 228) on three different days (on May 5, June 6, and June 15, 1998) and the B-200 King Air aircraft from NASA Langley Research Center was flown under TOPEX ground tracks on December 7, 1999, November 11, 1999 and December 29, 1999. These flights offered the first direct comparison between cross-correlation measurement from the delay-mapping receivers and existing instruments.

In addition, the B-200 aircraft was used in conjunction with the Space and Naval Warfare Systems Command (SPAWAR) Electro-Optical Propagation Assessment in Coastal Environments (EOPACE) Program, sponsored by the Office of Naval Research (ONR). These flight experiments took place off the coast of Duck, NC, on five different days (March 1, 5, 8, 11, and $12,1999)$ using a similar ground track. Surface truth data were available at three different locations: the Duck meteorological station (DUCN7) and two research buoys provided for this experiment. 


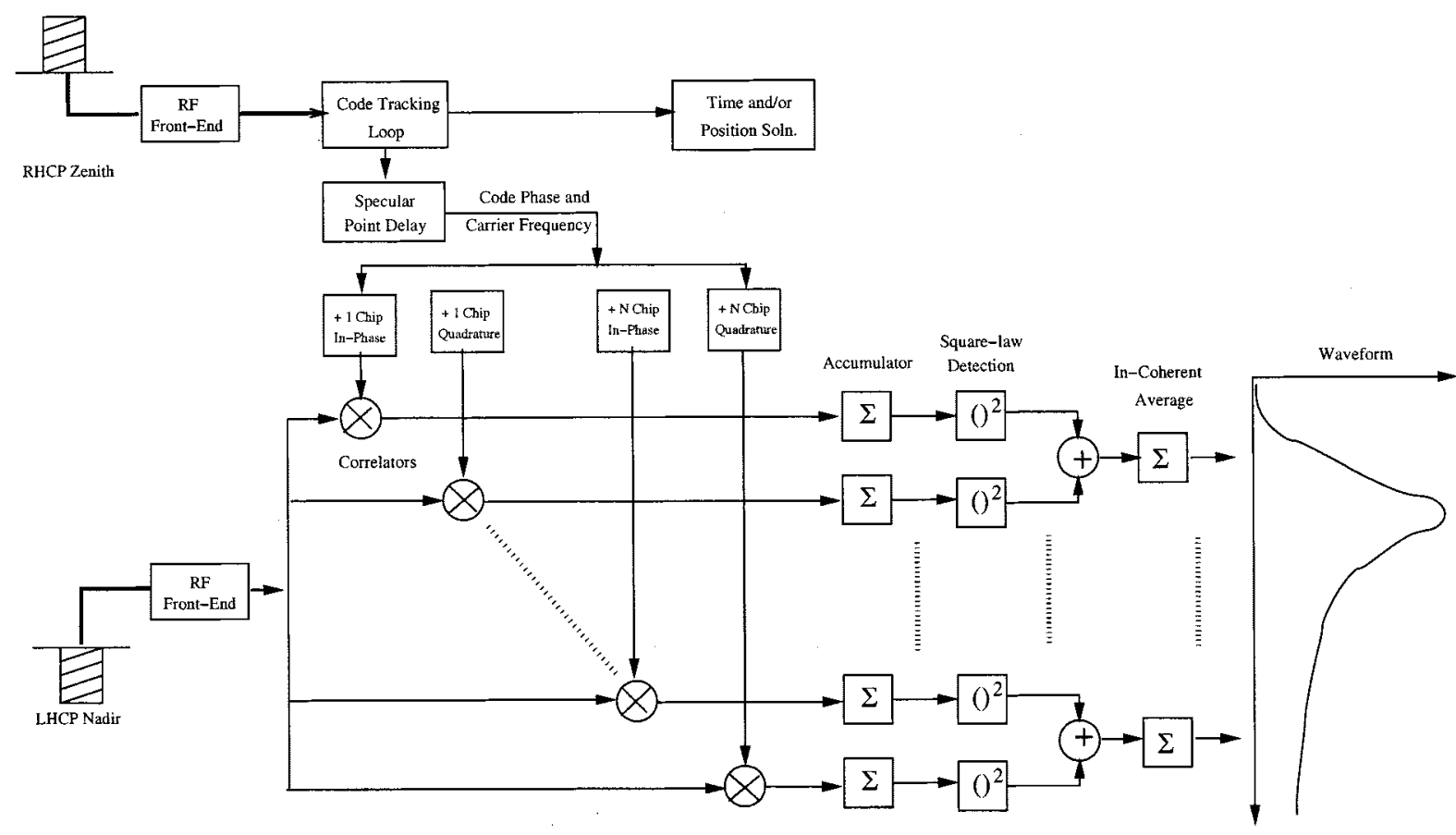

Fig. 1. Parallel delay mapping receiver (PDMR).

On August 22, 1998, the SDMR hardware was carried to altitudes in excess of $25 \mathrm{~km}$ on a balloon experiment conducted by the Virginia Space Grant Consortium (VSGC). This experiment collected data that first mapped the altitude dependence of the reflected signal waveform shape [11].

On all of the aircraft flights, an Ashtech Z-12 survey receiver was also carried. Differential corrections were used with postprocessing software to determine the aircraft flight path very accurately from this receiver. Precise GPS ephemerides from the National Geodetic Survey [12] were also obtained for the days of the flights.

\section{INDEPENDENT SOURCES OF DATA FOR COMPARISON}

The TOPEX radar altimeter on the TOPEX/Poseidon satellite essentially provides the sea surface height from the elapsed time in two way journey, from the satellite to the surface and back, of the radar pulse. In addition to giving estimate of sea surface height, it also gives significant wave height (SWH) from the slope of the leading edge of the returned pulse and wind speed over the ocean from the strength of the returned backscattered power. The scattering from the surface depends upon surface roughness, which in turn depends on wind speed. An altimeter provides the estimate of ocean surface wind speed from the measurement of normalized backscattering cross section $\sigma_{0}$ of the sea surface [13]-[16].

TOPEX computes $\sigma_{0}$ using the well-known radar equation [17]. It assumes that $\sigma_{0}$ is constant over the area for a spherically expanding short pulse incident on a smooth sea surface [15]. The $\sigma_{0}$ is computed from the ratio of the power received $\left(P_{r}\right)$ and the power transmitted $\left(P_{t}\right)$ modified by losses such as wave guide loss $L_{t}$ (i.e.,measured power is different from transmitted power), attitude loss $L_{\text {att }}$ (i.e., off-nadir pointing), and atmospheric loss $L_{a t m}$. These three loss terms, each greater than one, correct the received power. $\sigma_{0}$ is solved for using the following equation:

$$
\frac{P_{r} L_{t} L_{a t t} L_{a t m}}{P_{t}}=\frac{G^{2} c_{P} \lambda^{2} \sigma_{0}}{64 \pi^{2}\left(1+h / R_{e}\right) h^{3}}
$$

in which $G$ is the peak antenna gain, $c$ is the speed of light, $\lambda$ is the radar wavelength, $\tau_{P}$ is the radar pulse duration, $R_{e}$ the mean radius of Earth, and $h$ is the height above nadir point [18].

The wind speed measurements are derived using the model function provided by Witter and Chelton [19] (see also [20]). This function is an empirical relationship between wind speed and TOPEX-derived $\sigma_{0}$ values derived from some 240000 Seasat scatterometer measurements. TOPEX measured $\sigma_{0}$ are converted to wind speeds by a linear interpolation between tabulated values of $\sigma_{0}$ and wind speeds in the Seasat data base. In general, the radar altimeter gives wind speed with an accuracy of $2 \mathrm{~m} / \mathrm{s}$ in the range of 2 to $15 \mathrm{~m} / \mathrm{s}$. The lower and upper limits are due to the inherent measurement limitations associated with specular reflections [19].

\section{SCATTERING MODEL}

A theoretical model of GPS reflections in the near-specular forward direction has been derived from the geometric optics limit of the Kirchhoff approximation for the bistatic, rough-surface scattering problem [4] (see also, [21]). This model was used to generate the predicted shape of the cross-correlation waveform as a function of surface wind speed. These waveforms, approximated either with an exponentially decaying trailing edge slope (linear on a decibel scale) or with a series expansion, were then used in a parameter estimator to determine the optimal wind speed estimate given experimental waveform samples. 
The geometric optics model works well for a strong LHCP signal, which is of primary interest. It considers only quasispecular reflections from the large-scale (larger than several radio waves) components of the rough surface. The contribution from a small-scale roughness is insignificant for this type of polarization and at these angles. For a weak RHCP signal, this approximation does not work and the first-order small-slope approximation is needed, which accounts both for large- and small-scale surface components [22]. Therefore, the LHCP scattered signal (voltage) accumulated for an integration time of $T_{i}$ at the receiver position can be expressed by the integral taken over the mean sea surface

$$
Y(t, \tau)=T_{i} \int D(\vec{\rho}) \Lambda\left[\tau-R_{0}(\vec{\rho})-R(\vec{\rho})\right] g(\vec{\rho}, t) d^{2} \rho
$$

Here, $D(\vec{\rho})$ is the initial footprint of the receiver antenna in terms of an amplitude. Functions $R_{0}(\vec{\rho})$ and $R(\vec{\rho})$ are distances from the transmitter and the receiver, respectively, to some point on the uneven surface. The polarization sensitive function $g(\vec{\rho}, t)$ describes propagation and scattering processes in the geometric optics limit of the Kirchhoff approximation. For aircraft and balloon altitudes, the Earth's curvature is neglected. The Doppler effect caused by relative motion of the transmitter, receiver, and Earth's surface can be considered as uniform within the surface area of interest. For the low gain hemispherical antennas used on all of these experiments, the antenna gain $D(\vec{\rho})$ can be assumed to be uniform over the glistening surface. The function $g(\vec{\rho}, t)$ is expanded into surface distances in (5), and the scattering cross section in (8).

The average power following correlation $\left(Y^{2}(\tau)\right)$ as a function of a relative delay $\tau$ is obtained by squaring inphase and quadrature components of the signal voltage and then averaging over the accumulation time $T_{a}$. Assuming that integration over the accumulation time $T_{a}$ is equivalent to averaging over a statistical ensemble of surface elevations and making some additional simplifications we arrive at the following equation:

$$
\begin{aligned}
\left\langle\left|Y\left(t_{0}, \tau\right)\right|^{2}\right\rangle & \equiv \frac{1}{T_{a}} \int_{t}^{t+T_{a}}|Y(t, \tau)|^{2} d t \\
& =T_{i}^{2} \int \frac{D^{2}(\vec{\rho}) \Lambda^{2}\left(\tau-R_{0}-R\right)}{4 \pi R_{0}^{2}(\vec{\rho}) R^{2}(\vec{\rho})} \sigma(\vec{\rho}) d^{2} \rho .
\end{aligned}
$$

Here, distances $R_{0}$ and $R$ have meaning of distances from the transmitter and the receiver, respectively, to some point on the mean sea surface. This type of equation is known as a bistaticradar equation. Equation (5) is a more precise form of (2) as can be shown by use of the mathematical identity

$$
\Lambda^{2}\left(\tau-R_{0}-R\right)=\int \delta\left(\eta-R_{0}-R\right) \Lambda^{2}(\tau-\eta) d \eta
$$

Upon substituting (6) into (5) and comparing it with (2) we obtain

$$
p(\eta)=T_{i}^{2} \iint \frac{D(\vec{\rho}) \delta\left(\eta-R_{0}-R\right)}{4 \pi R_{0}^{2}(\vec{\rho}) R^{2}(\vec{\rho})} \sigma(\vec{\rho}) d^{2} \rho .
$$

The $\delta$-function under the integral symbolizes a contribution to the received power from an infinitely thin elliptic annulus on the surface. The full power is a result of a convolution $p(\eta)$ with $\Lambda^{2}(\tau-\eta)$

Factor $D^{2}$ can be taken out of the integral in (5) for the case of a low-gain antenna. The function $\Lambda^{2}$ occupies the area of an elliptic ring expanding with $\tau$ and only accumulating signal power from the elliptic ring (also known as annulus zone). Because of this, the integration in (5) includes only the part of a surface, which satisfies the condition $\left|\tau-R_{0}-R\right|<\tau_{C H I P}$. The quantity $\sigma(\vec{\rho}) \equiv \sigma(\vec{q})$ is the normalized bistatic scattering cross section of the ocean surface. This is a function of the surface slope at the position $\vec{\rho}$ on the surface. This slope is conveniently describe by the vector $\vec{q} \equiv \vec{\kappa}(\vec{\rho})-\vec{\kappa}_{0}(\vec{\rho})$, in which $\vec{\kappa}_{0}$ and $\vec{\kappa}$ are wave vectors of incident and scattered waves, respectively. $\sigma$ can be represented as

$$
\sigma=\pi|\mathcal{R}|^{2}\left(q / q_{z}\right)^{4} P\left(-\vec{q}_{\perp} / q_{z}\right) .
$$

Here, (8) is limited to a regime of so-called diffusive scattering from sea surface (or large values of the Rayleigh parameter). The value of $\sigma$ depends on a complex Fresnel coefficient $\mathcal{R}(\theta) . \mathcal{R}(\theta)$ in turn depends on the polarization, the complex dielectric constant of sea water, and the local elevation angle $\theta$. $P\left(-\vec{q}_{\perp} / q_{z}\right)$ in (8) is the probability density function (PDF) of surface slopes. These slopes characterize only the large-scale part of the ocean surface. The most probable orientation of slopes on the ocean surface is parallel to the horizontal plane $(z=0)$. Thus, the PDF has a maximum for slope of zero, and the bistatic cross section $\sigma$ has a maximum at nominal specular direction with respect to the mean sea surface.

The probability distribution of surface slopes is dependent upon the wind speed and direction. Statistics of these slopes are assumed to be Gaussian with wind-dependent upwind and cross-wind variances $s_{u}^{2}, s_{c}^{2}$. These variances can be estimated from data obtained in the Cox and Munk optical measurements [9] for the ocean surface with slicks that has suppressed gravitycapillary and capillary waves shorter than $30 \mathrm{~cm}$. A more convenient way is to obtain these variances from empirical wavevector spectra $\Psi(\vec{\kappa})$ of full surface elevations by integrating it over bidirectional wave numbers smaller than a dividing parameter $\kappa_{*}$ of the two-scale model (see, e.g., [23]). We choose $\kappa_{*}=$ $2 \pi \sin \theta_{0} / 3 \lambda$ which is usually considered a reasonable number, where $\lambda$ is the radio wave length, and $\theta_{0}$ is a nominal GPS elevation angle

$$
s_{u, c}^{2}=\int_{\kappa \leq \kappa_{*}} \kappa_{u, c}^{2} \Psi(\vec{\kappa}) d^{2} \kappa .
$$

Here, we are using an empirical spectral model for $\Psi(\vec{\kappa})$ proposed by Elfouhaily et al. [24]. This model includes dependence on fetch, which allows us to consider fetch-limited or nonfully developed seas. Variances calculated from this spectrum agree with Cox and Munk optical measurements [9] of these parameters. There is no agreement among the ocean remote sensing community about which model should be considered as the best one. For example, authors of the paper [25] expressed criticism toward the capillary-gravity part of the spectral model by Elfouhaily et al. However, in this application, that part of the spectrum plays a minor role since quasispecular mechanism involves mostly the large-scale part of the ocean roughness. 


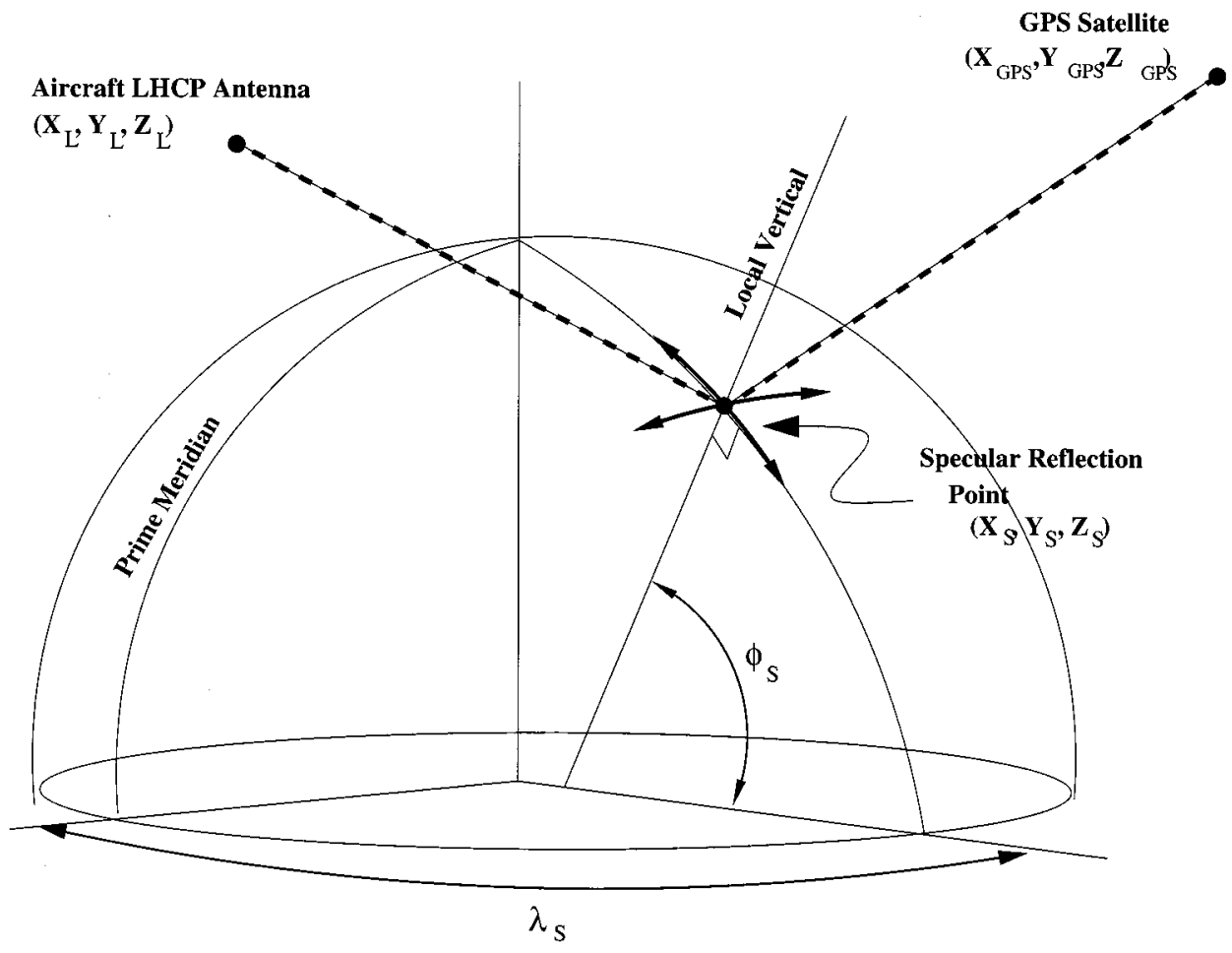

-1........ Reflected Ray Path

Fig. 2. Geometry used to determine the location of the specular point and the reflected ray path length (WGS-84 ellipsoid assumed).

The mechanization of this analytical model, as part of the parameter estimation of surface wind speed, is described in detail in Section VI.

\section{PostPROCESSING}

Postprocessing was performed on the raw, or level 0 data from the receiver which accomplishes two basic functions: determining the location of the specular reflection point on the surface of the ellipsoidal Earth, and re-alignment of each (1/2 code chip spaced) correlator sample in code delay relative to the specular point. This was done to precisely reference the measurement to the location of ground truth (the location of the specular point can be several kilometers from the aircraft ground track) and to map the waveform samples with finer than $1 / 2$ chip precision.

This realignment was performed through use of the navigation data from the aircraft and the NGS ephermides. Timing of the measurements was either provided from the GPS solution (in the case of four or more direct satellites tracked in the SDMR), or from a single satellite in the case of the PDMR. The true path length difference of a specular reflection from the surface of the WGS-84 ellipsoid was computed by minimizing the total path length of a reflected signal as in (10), shown at the bottom of the page.

The Earth-centered Earth-fixed coordinates of the LHCP antenna phase center $\left(X_{L}, Y_{L}, Z_{L}\right)$ were obtained from the differentially corrected Ashtech flight path (assuming level flight). The GPS satellite coordinates ( $X_{\mathrm{GPS}}, Y_{\mathrm{GPS}}, Z_{\mathrm{GPS}}$ ) were obtained from the NGS ephemerides. The coordinates of the specular point $\left(X_{S}, Y_{S}, Z_{S}\right)$ were found by numerically minimizing $\rho_{L}$ through varying the geodetic latitude and longitude, $\phi_{S}$ and $\lambda_{S}$ of a specular point which was assumed to be fixed to lie on the WGS-84 ellipsoid. The geometry of the specular point and reflected ray path length is illustrated in Fig. 2. Further details of these coordinate systems can be found in [26].

The difference between this geometric location of the specular point and the stored code phase information from sampling the code epoch for each correlator was used to compute the relative delay between each power sample and the specular point. The correlation power was then normalized such that it had a constant total area of one half code chip through dividing each power sample in a given waveform by the total integrated power in all range bins for that waveform. This removed changes in the intensity of the incident radiation and was also found to

$$
\begin{aligned}
\rho_{L}=\sqrt{\left(X_{L}-X_{S}\left(\phi_{S}, \lambda_{S}\right)\right)^{2}+\left(Y_{L}-Y_{S}\left(\phi_{S}, \lambda_{S}\right)\right)^{2}+\left(Z_{L}-Z_{S}\left(\phi_{S}, \lambda_{S}\right)\right)^{2}} \cdots \\
+\sqrt{\left(X_{\mathrm{GPS}}-X_{S}\left(\phi_{S}, \lambda_{S}\right)\right)^{2}+\left(Y_{\mathrm{GPS}}-Y_{S}\left(\phi_{S}, \lambda_{S}\right)\right)^{2}+\left(Z_{\mathrm{GPS}}-Z_{S}\left(\phi_{S}, \lambda_{S}\right)\right)^{2}} .
\end{aligned}
$$




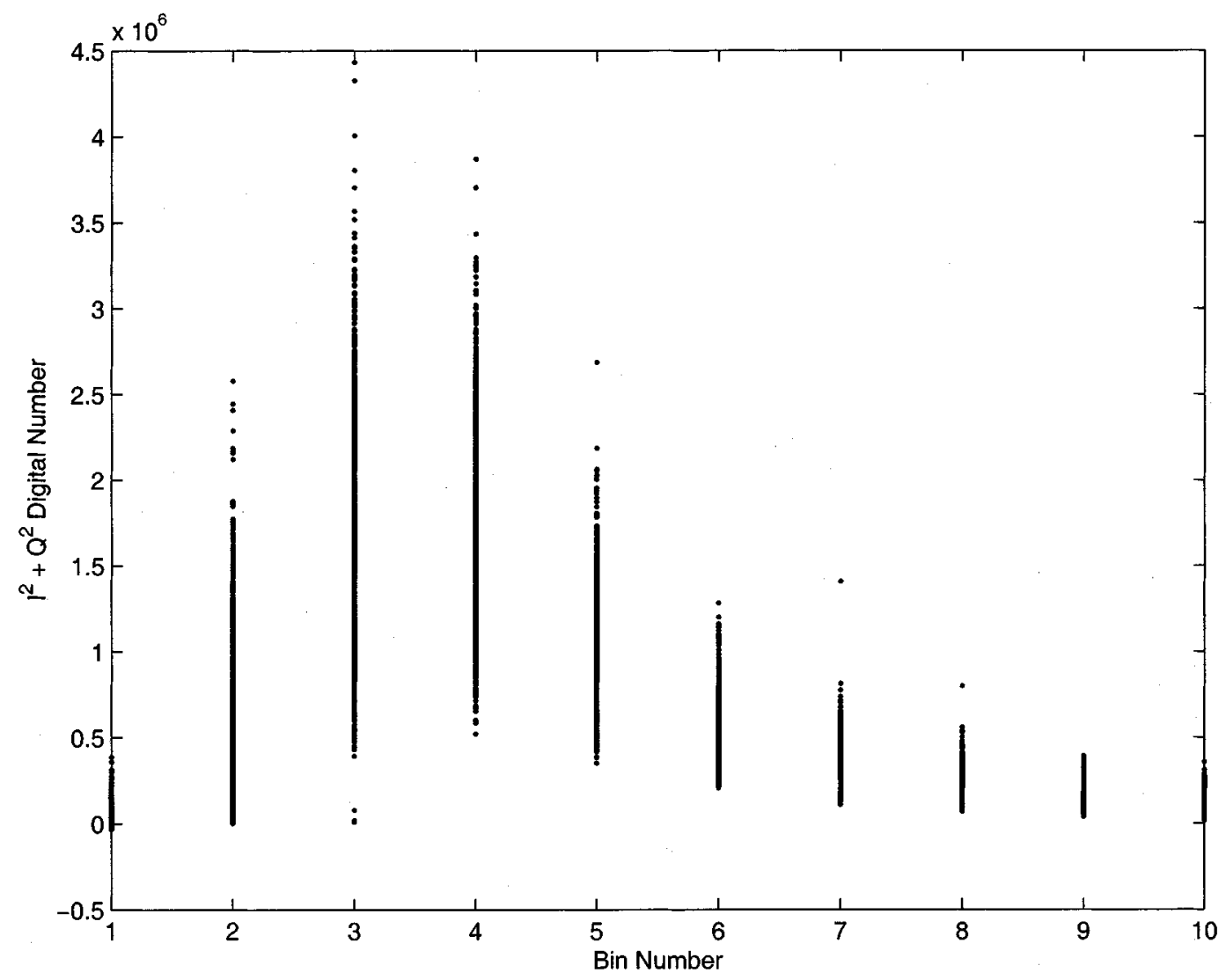

Fig. 3. Raw waveform data from PDMR correlators.

produce results with the lowest variance. This effectively eliminated the need to calibrate the lower channel correlation values with a physically meaningful measurement of reflected power and eliminated many uncalibrated effects such as aircraft attitude motion and the antenna gain pattern. An example of the raw waveform data extracted from the receiver is given in Fig. 3. The normalization to constant area, and alignment relative to specular point is illustrated in Fig. 4.

Notice that for this example, there appears to be two waveforms superimposed on each other. The correction of this effect is described later in this paper.

The result of postprocessing is a standard set of "level 1a" data. This consists of waveform measurements of the incoherent power $Y_{k}^{2}\left(\tau_{i}\right)$ at specific code delays $\tau_{k, i}$. For each $k$ th waveform sample (taken at a $1 \mathrm{~Hz}$ rate for the SDMR and a $10 \mathrm{~Hz}$ rate for the PDMR) auxiliary information giving the specular point location $\left(\phi_{S_{k}}, \lambda_{S_{k}}\right)$ and the GMT of the measurement are also tabulated.

These waveform samples are then provided for the retrieval techniques which match the geometry of the waveform shape to theoretical model predictions to estimate surface wind speed. In this paper, summaries are provided for two of these techniques.

\section{Estimation TEChNiQues}

\section{A. Wind Speed Estimation Using the Waveform Trailing Edge}

The first retrieval technique includes the generation of sets of modeled waveforms and the normalized power versus time delay with respect to the nominal specular point, for various wind speeds. The trailing edge of the waveform taken in a logarithmic scale was used for the estimation, being the most sensitive part to surface wind speeds.

The measured signals were normalized by the integral power observed in delay bins within the expected range of the glistening zone. Measurements showed that there is no strong signal correlation between the top and bottom channels. The total reflected power is not expected to depend on surface statistics and can be used as a normalization factor. To smooth these data, a 150 s sliding average was applied to the raw reflected GPS measurements. As described above, an adaptive signal normalization, which generates a constant area waveform was used to cancel out the uncalibrated effects of antenna gain, aircraft attitude motion, and difference in cable loss and front-end gain between the direct and reflected signals. These data were used at the discrete half-code delays (corresponding to $150 \mathrm{~m}$ in range resolution) shown in Fig. 3. An approximate value for the noise floor was determined experimentally from the average correlation power in the last four delay bins. At low altitudes it was assumed that the power in the waveform at such long delays has decayed to a negligible amount. In contrast to the earliest bins, only the first one is expected to record noise only. Occasional misalignments of the correlator will also produce reflected power in the first bin. This effect is addressed in section B. The trailing edge slope on a decibel scale was estimated with a least squares fit of a straight line to the measured waveforms between 5 and $20 \mathrm{~dB}$. This slope compared to an straight-line (on a dB scale) approximation to the trailing edge 


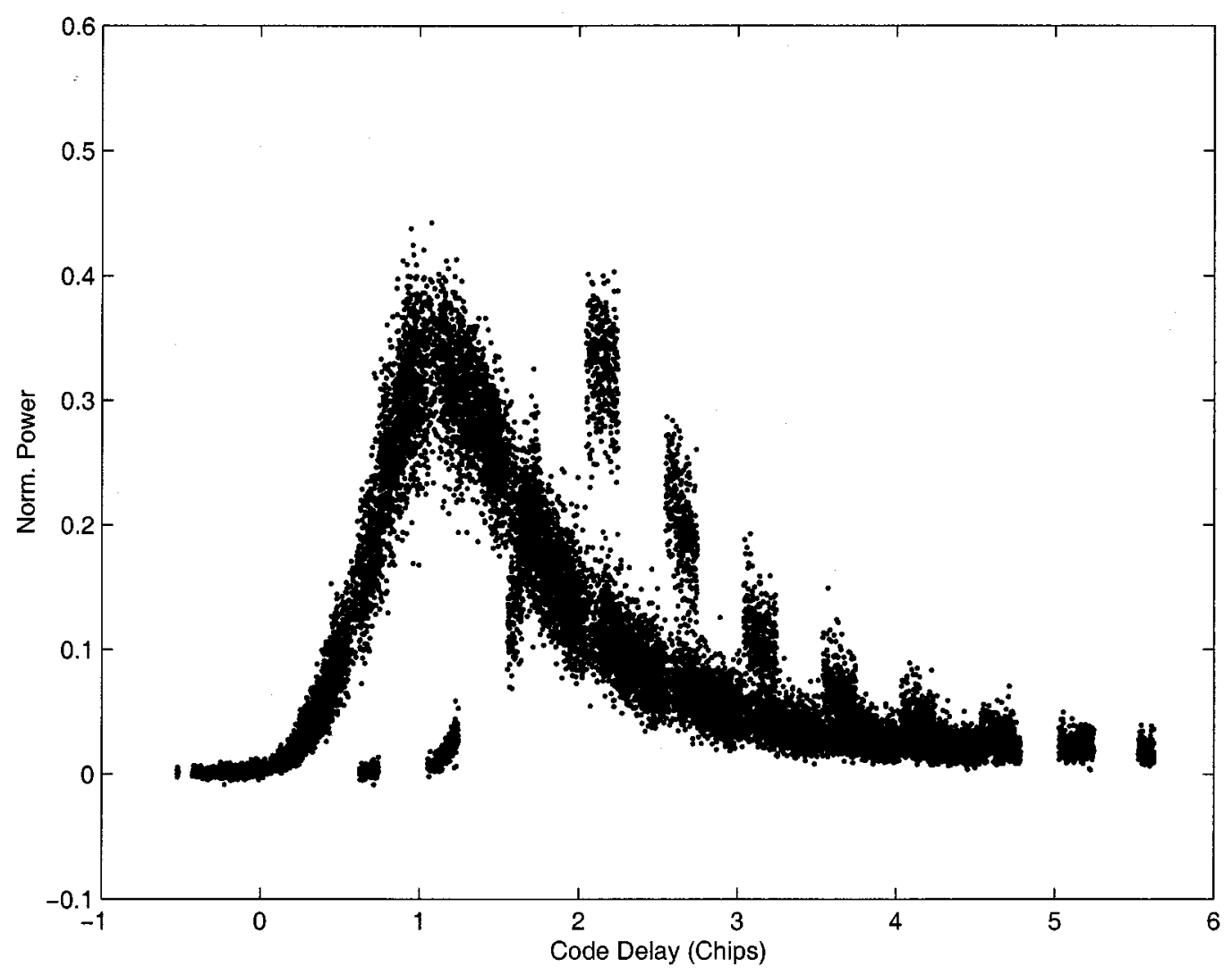

Fig. 4. Normalized and georeferenced correlation data. Note the superposition of two waveforms.

of the theoretical model described in Section IV. This approximate trailing edge slope was generated using various incremental wind speeds. The estimated wind speed was obtained by interpolating the measured slopes between the modeled ones.

The TOPEX-altimeter over-flight covered a wide range of wind speeds ranging from $0.5 \mathrm{~m} / \mathrm{s}$ to $8.7 \mathrm{~m} / \mathrm{s}$ within a ground distance of about $40 \mathrm{~km}$. After processing the data, the TOPEXindicated geographic area was matched with the location of GPS measurements.

We have found the trailing edge of the waveform to be very stable between 5 and $20 \mathrm{~dB}$, therefore we used that region to routinely infer surface wind speeds. Fig. 5 shows this dependence. On this figure the normalized power in bins numbered 0 to 5 (lower number at shorter delays) is plotted as a function of windspeed showing the greatest sensitivity to wind speed in the higher numbered bins.

For every TOPEX wind speed measurement, the trailing edge slope was estimated using the measured waveforms between 5 and $20 \mathrm{~dB}$ and the theoretical model using various incremental wind speeds. The estimated wind speed was obtained by interpolating the measured slopes between the modeled ones. In Fig. 6, we displayed both the TOPEX measured wind speeds and our estimated ones using GPS data from two different satellites: PRN15 and PRN19. One standard deviation error bars were also displayed for the measured wind speeds. The figure shows that the error bars are larger for higher wind speed. This is due to the fact that for higher wind speeds the separation between two waveform slopes, corresponding to two separate wind speeds, are smaller than the separation between two wind speeds at

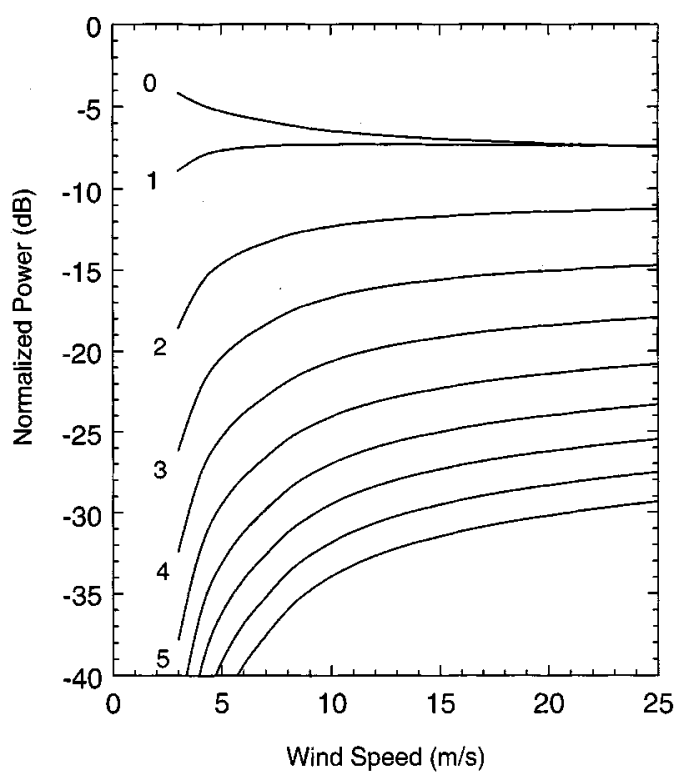

Fig. 5. Normalized power in several "bins" as a function of surface wind speed, indicating the strongest sensitivity is in the higher numbered bins. (i.e., on the waveform trailing edge).

smaller wind speeds. Furthermore, the figure indicates that wind speeds estimated independently using the two satellites show good agreement with the TOPEX-measured wind data.

These retrievals first demonstrated qualitatively the potential to estimate wind speed from the reflected GPS signal. Deviations of measured waveform shape from the theoretical models, 


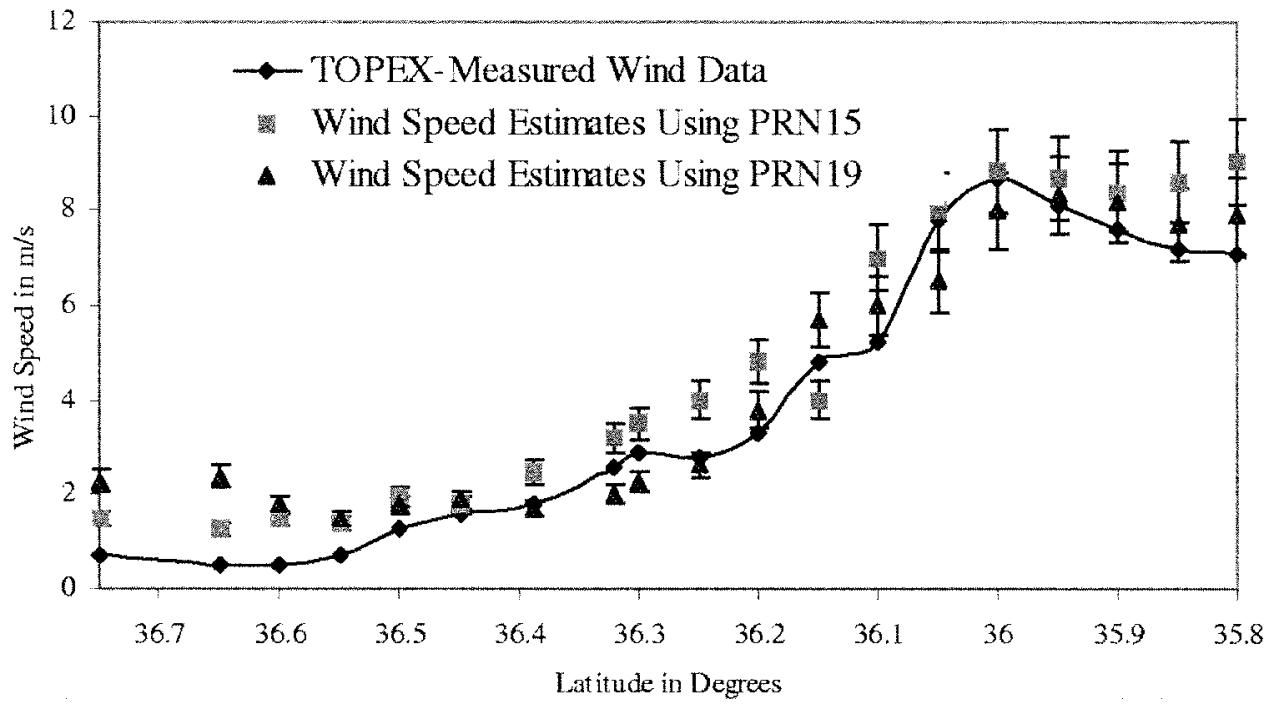

Fig. 6. Estimated wind speed using PRN15 and PRN19 and TOPEX ground truth. Error bars indicate one standard deviation.

which were shown in some of these plots, could be attributed to the following effects: using a measured noise floor, which may contain reflected signal power; binning data within half-code chip increments; and applying a linear least squares estimation to logarithmic data (which could introduce a bias in the estimate). Subsequent improvements in the wind speed estimation algorithm took into account additional information available from the receiver to reduce these effects.

\section{B. Series Approximation of Waveform Shape}

In general, the waveform $Y^{2}(\tau)$ will have some functional dependence on the following geophysical parameters: wind speed $\left(U_{10}\right)$, wind direction $(\vec{U})$, significant wave height $(\mathrm{SWH})$, and inverse wave age $(\Omega)$, and the frequency of down conversion prior to correlation $\left(f_{D}\right)$.

$$
Y^{2}(\tau)=f\left(\tau, f_{D}, U_{10}, \vec{U}, \Omega, \mathrm{SWH}\right) .
$$

Equation (11) summarizes the input to output relationship produced from the following application of the theoretical model described in Section IV.

- Given $U_{10}$, assume SWH $=0, \Omega=0.84$, the scattering plane is in the upwind direction, and $f_{D}$ is the same as the frequency derived from tracking the direct GPS signal.

- Generate the wave spectrum $\Psi(\vec{\kappa})$ from the Elfouhaily model [24].

- Integrate this spectrum, as in (9), up to wave numbers of $\kappa_{*}=2 \pi \sin \theta_{0} / 3 \lambda$. This produces values for the upwind and crosswind mean square slopes, $s_{u}^{2}, s_{c}^{2}$.

- Generate a Gaussian PDF using $s_{u}^{2}, s_{c}^{2}$, as seen earlier.

- Compute the scattering cross section from (8) for points within the glistening surface.

- Integrate the scattered signal over the surface for different values of the code delay $\tau$ using (5). This produces the waveform $Y^{2}(\tau)$ in (11).

Each of the parameters in (11) were considered, and it was shown that the wind speed was expected to be the predominant effect on the shape of the waveform for high-elevation satellites. First, to compare the sensitivity to wind direction and to the

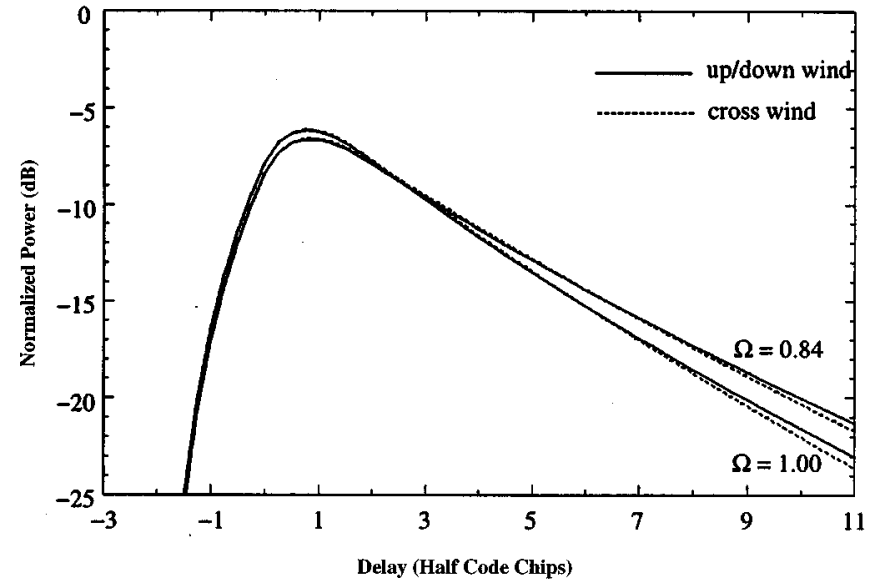

Fig. 7. Sensitivity of waveform shape to wind direction and inverse wave age

assumed value for inverse wave age $\Omega$ and wind direction, see Fig. 7. In this figure, the receiver altitude was $12 \mathrm{~km}$, the GPS elevation angle was $69^{\circ}$, and wind speed $U_{10}$ was $5 \mathrm{~m} / \mathrm{s}$.

This figure indicates that a small sensitivity to wind direction becomes apparent at signal levels below approximately -20 $\mathrm{dB}$. Therefore, the upper part of waveforms (above approximately $-20 \mathrm{~dB}$ ) where measurements will have a higher SNR can be used for wind speed retrieval regardless of the wind direction. It was experimentally observed that the wind direction becomes important for satellites with lower elevations (below 30 to $40^{\circ}$ ). This was apparent from larger errors when retrievals were attempted estimating wind speed only from those satellites. Second, comparisons of these curves with the data from Fig. 7 show that the curve obtained for fully developed seas $(\Omega=0.84)$ is wider than that for nonfully-developed seas $(\Omega=$ 1.00). This will result in lower than expected wind speeds if the fully developed seas condition is not met. This effect was not observed during the experimentation presented in this paper. However, we cannot insist that during all experiments we indeed have had fully-developed seas since there were not any independent measurements of parameter $\Omega$ available. One could imagine a situation when an increase in $\Omega$ is compensated by a presence 


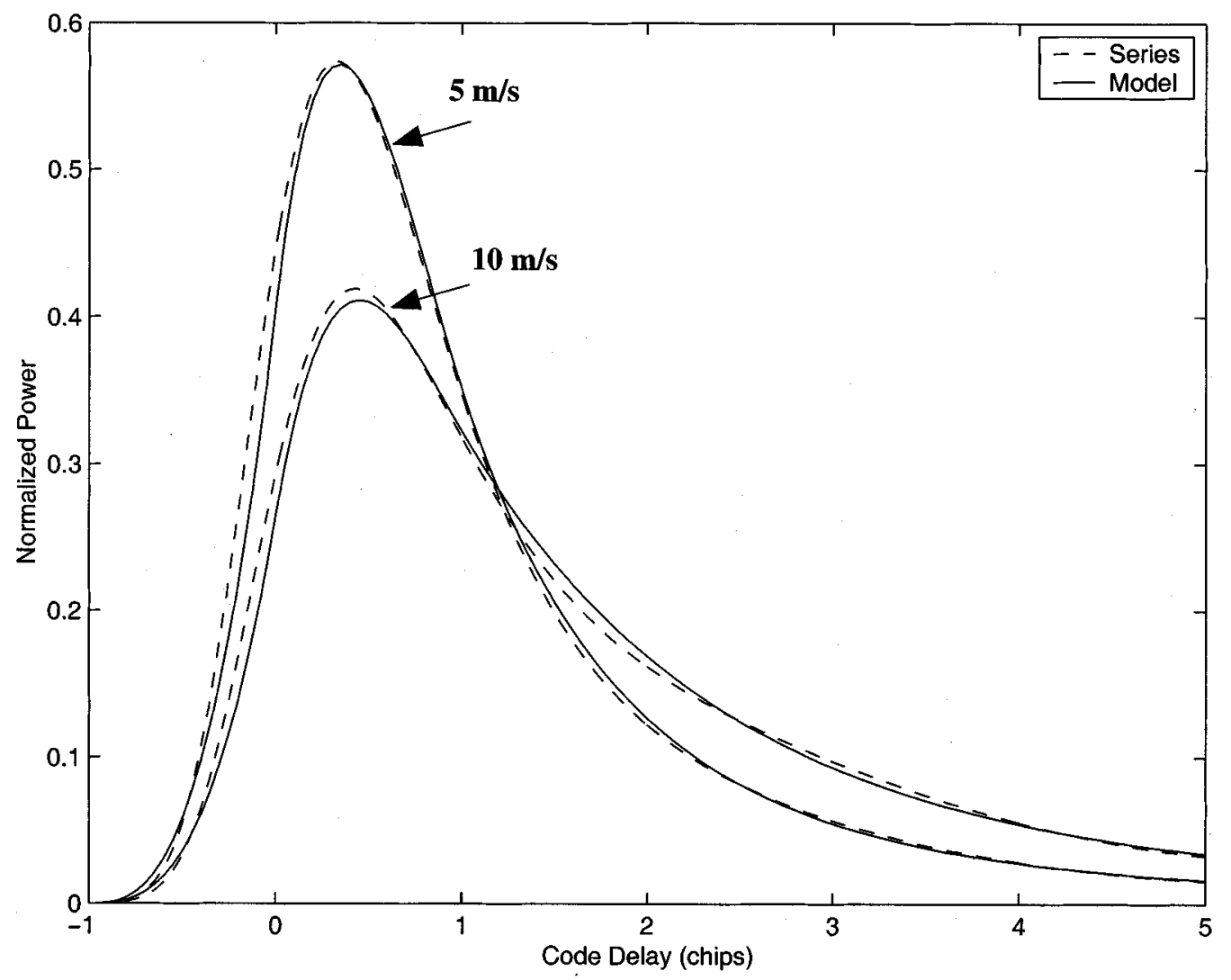

Fig. 8. Comparison of waveform shape to series approximation.

of swell that adds more to the slope variance. This is possible when winds are weak $(5 \mathrm{~m} / \mathrm{s})$ and therefore the wind induced slopes are relatively small, so swell may be of importance. For stronger winds (higher than $10 \mathrm{~m} / \mathrm{s}$ ), the influence of swell is less important.

The variation of surface height will also cause some portion of the signal to arrive at the receiver with a path length shorter than that through the specular point. At most, the difference in path length as a result of surface height variation will be on the order of meters. Following convolution of this signal with the $\Lambda^{2}$ autocorrelation function of the PRN code (which has a width of approximately $600 \mathrm{~m}$ ), the postcorrelation waveform shape will not show any significant dependence on the height variation.

Only the magnitude of wind speed $\left(U_{10}\right)$ was considered in this study. Anisotropic surface statistics [4], or skewed nonGaussian probability density functions [8] will make the waveform shape weakly dependent upon wind direction. It has not been determined if this dependence is strong enough to obtain any useful retrievals of wind direction using waveform data from a single satellite alone. Recently, however, an estimation of both speed and direction, using simultaneous processing of the waveforms from two satellites, was presented [27].

The practical computation of the cross-correlation in (5) requires a double integral over the surface. This cannot be analytically inverted. It was best to use an optimal parameter estimator on an overdetermined set of measurements. This required that this integral be computed for each measurement point, on multiple iterations to update the parameter estimator. Performing the complete integration for each point during every integration step called by the estimator would have been computationally intensive. For that reason, this integration was approximated by a finite series expansion in terms of wind speed $\left(U_{10}\right)$ and code delay $(\tau)$ having the following form:

$$
Y^{2}(\tau) \approx S \exp \left[\sum_{i=0}^{N} \sum_{j=0}^{M} a_{i, j} U_{10}^{j}\left(\tau-\tau_{0}\right)^{i}\right]
$$

An eighth order series of this form, in which the coefficients $a_{i, h}$ were determined numerically to best fit the complete model over a range of wind speeds from $1.75 \mathrm{~m} / \mathrm{s}$ to $21.75 \mathrm{~m} / \mathrm{s}$ and assuming a constant wind direction, was found to make a very good approximation to the shape of the waveform. It should be noted that the observation model defined by (2) or (5) involves the convolution of two functions, one of which $\left(\Lambda^{2}(\tau)\right)$ is ideally discontinuous at $\tau=0$. Therefore, approximating the waveform shape with a continuous function is expected to be difficult for cases in which the waveform becomes "sharp." (i.e., at low altitudes or low wind speed and near the peak.) The comparison between this series approximation, and the output of the model for a very narrow waveform and a very wide one is shown in Fig. 8.

This series approximation, in which the set of $a_{i, j}$ were precomputed for the average altitude and GPS satellite elevation during a period of data collection, was used as the observation equation and three parameters: wind speed $U_{10}$, delay of the peak of the waveform from the computed specular point, $\tau_{0}$, and the scale factor $S$ were then estimated using a 


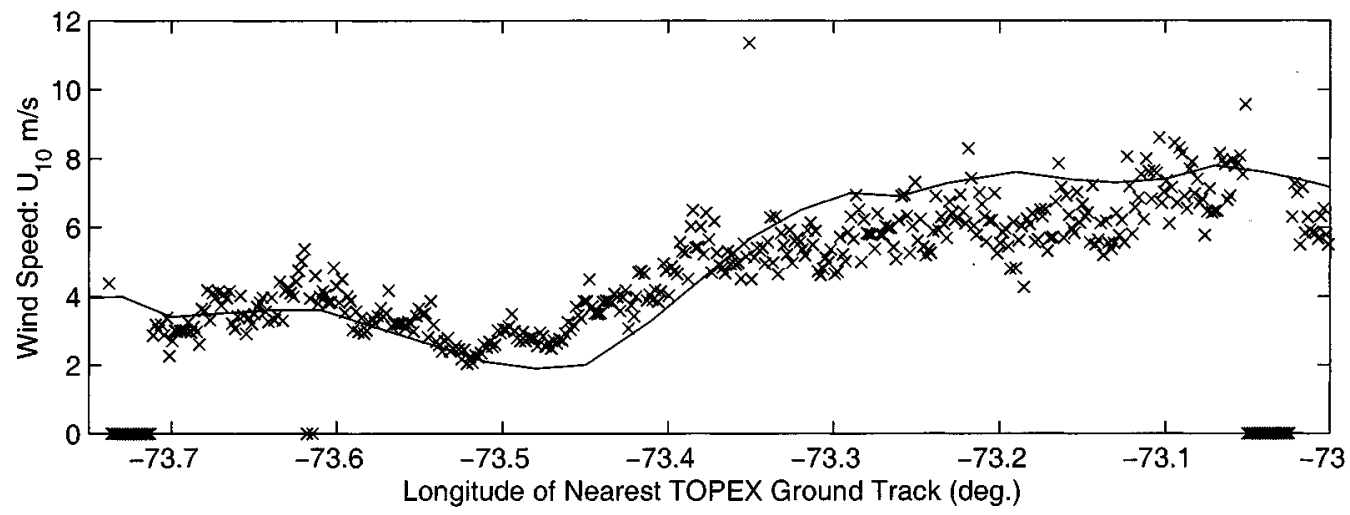

Fig. 9. Example time history of $U_{10}$ estimate: June 15, 1998 TOPEX Underflight, Satellite PRN 3.

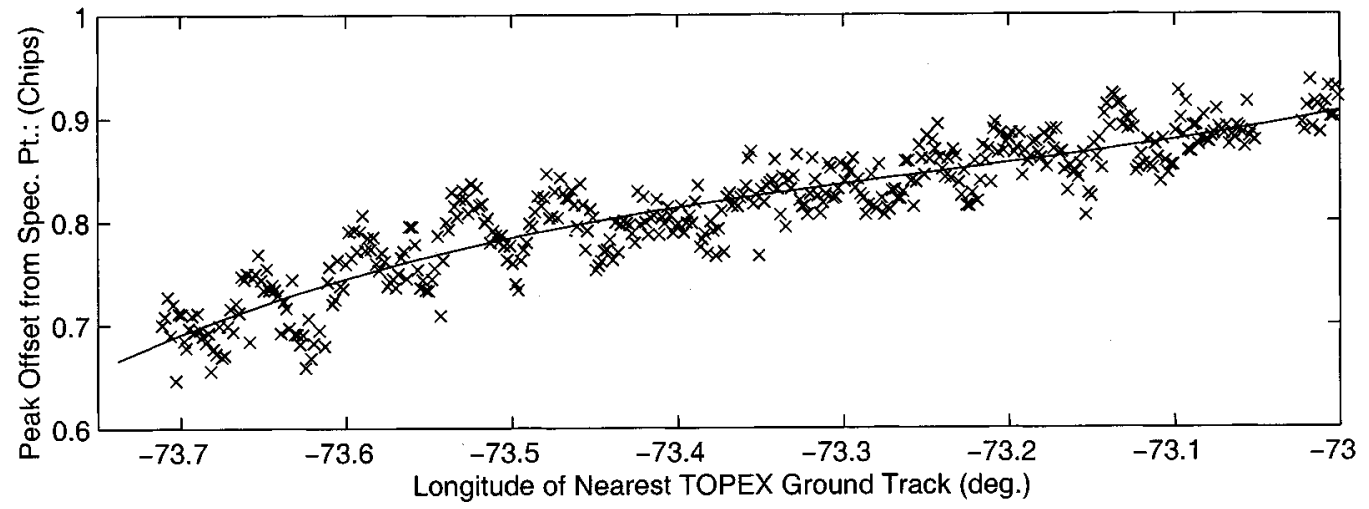

Fig. 10. Example time history of $\tau_{0}$ estimate: June 15, 1998 TOPEX Underflight, Satellite PRN 3.

nonlinear least squares method to batch segments of waveform data $\left(\tau_{k, i}, Y_{k, i}^{2}\right)$. The least squares minimization problem was solved through a large-scale optimization subroutine provided in the optimization toolbox in MATLAB [28]. This algorithm uses an interior-reflective Newton Method [29], [30]. No weighting of the data was assumed. In other words, it was assumed that the uncertainty was the same for any sample of a waveform and that each sample was independent of any other taken either at a different delay in the same waveform or at a different time. Visual inspection of Fig. 4 suggests that variance in signal power does not change significantly for different delays along the trailing edge (where the measurement is most sensitive). However, the convolution in (2) will result in strong correlation between adjacent samples which are spaced closer than one code chip apart. Future work therefore should be directed to incorporating a data covariance matrix into this parameter estimation.

A constant noise floor, determined using the theoretical value set by the AGC prior to digital sampling, was subtracted from all correlation measurements before the constant area normalization was performed.

Although each waveform sample was scaled to have a constant area of one half chip, the scale factor $(S)$ was found to improve the estimation accuracy. The reason for this was postulated to be: a finite number of delay bins do not record the complete waveform because portions of the trailing edge often extend beyond the range of these bins. The scale factor therefore accounts for the fact that the measured waveform, once scaled to be constant area, has slightly larger area than that predicted by theory. Using the parameter $\tau_{0}$ effectively calibrated the relative delay between the direct and the reflected signal path lengths. Figs. 9 and 10 show the time history of the parameters $U_{10}$ and $\tau_{0}$ for a typical segment of reflected GPS data collected under the TOPEX ground track. The wind speed estimate is plotted in Fig. 9. For comparison, the TOPEX wind speed obtained along the same ground track is plotted as the solid line on this figure. Fig. 10 plots the estimates of $\tau_{0}$ for the same segments of data. The random error in $\tau_{0}$ could also be interpreted as rough estimate on the practical accuracy achievable using the reflected GPS signal as an altimeter (as proposed in [2]). The time history of $\tau_{0}$ in Fig. 10 shows a long-term trend. Once this trend, approximated as a third-degree polynomial is subtracted, the standard deviation of the residual is approximately 0.02 chip (7.4 $\mathrm{m})$. The polynomial trend is plotted on Fig. 10 as well.

Three anomalies were not accounted for in these signal models. These were identified and corrected before the data were processed by the least squares estimator. Editing the data in this manner was found to improve the performance of this estimator and removed most of the outliers in the wind speed estimates.

First, prior to processing of a batch of data, the total power in all delay bins was computed and if this fell below a threshold, then the complete waveform was rejected. This eliminated measurements made over land, and time in which the receiver had not acquired a strong reflected signal. This threshold was set to a value that would correspond to an average signal to noise ratio 


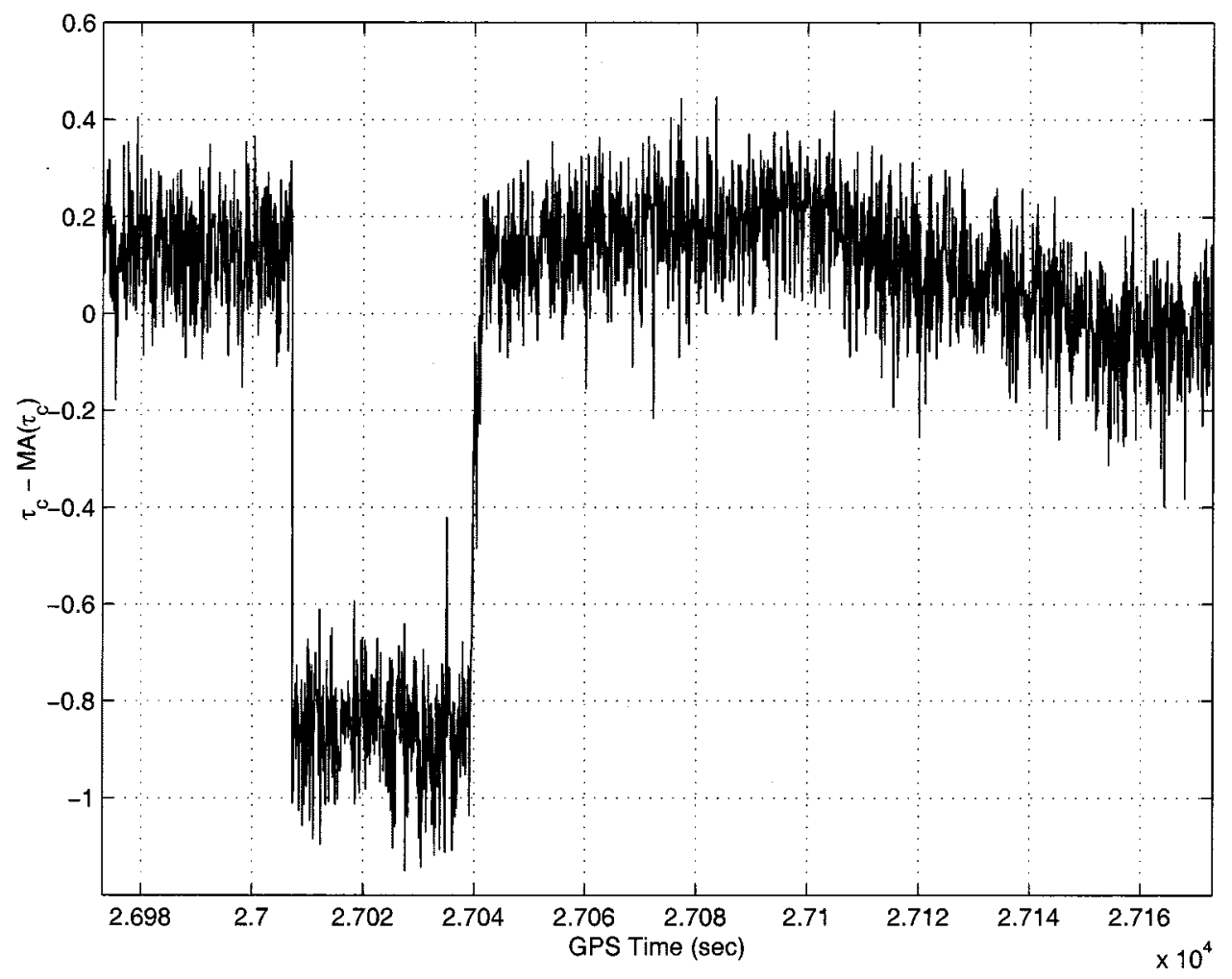

Fig. 11. Waveform centroid identifying slip of one code chip.

of approximately $4 \mathrm{~dB}$ per correlator. Second, occasional slips by an integer number of half code chips in the spacing of the delay bins was observed (as demonstrated by the two superimposed waveforms on Fig. 4).

This anomaly was detected by searching for discontinuities in the waveform centroid

$$
\tau_{c}=\frac{\sum_{i}^{M} Y^{2}\left(\tau_{i}\right) \tau_{i}}{\sum_{i}^{M} Y^{2}\left(\tau_{i}\right)} .
$$

$\tau_{c}$ was computed at each time step from every $i$ th pair of power and delay, in which the power exceeded four times the noise floor standard deviation. The time history of $\tau_{c}$ at each sample was then compared against the moving average of $\tau_{c}$ using a window of $100 \mathrm{~s}$. When the instantaneous $\tau_{c}$ differs from more than one chip, a correction of $\left|\tau-\overline{\tau_{c}}+0.5\right|$ is applied. This waveform slip on Fig. 4 is clearly evident in the plot of the centroid in Fig. 11.

This correction is illustrated in Fig. 12. An improved receiver design (to eliminate this failure at the source) could reduce these cases.

The third effect identified was another receiver artifact in which the leading edge of the waveform extended earlier than the first correlator. In these cases, there was correlation power in the earliest correlator power bin. Attempts to fit the function defined in (12) to this situation resulted in a wider than required waveform shape and consequently a higher wind speed. Individual waveforms in which this correlation power in the first bin exceeds a threshold (set experimentally, but would require approximately ten standard deviations increase in the noise value) are deleted from the ensemble. Although most of the information about the surface slope statistics is contained in the trailing edge of the waveform, this method was found to be very sensitive to the requirement that the power earlier than -1 chip return to zero. At present, all that was done to correct for this effect is to delete the waveform sample from the ensemble altogether. It may be possible to include zero-valued power measurements at time delays earlier than the start of the measurements so as to force the curve fitting algorithm to converge to zero for delays earlier than -1 code chip relative to the specular point. This correction would be justified because no reflected power could be received earlier than the specular point and therefore no correlation could exist earlier than 1 chip prior to the specular point. Methods described earlier which use only the trailing edge data would not experience this anomaly.

Finally, a residual monitoring process is used in which a threshold is set on the sum square of residuals in one waveform. This was computed from the difference between the observed and computed power following the least squares estimate for the $k$ th waveform:

$$
r_{k}=\sum_{i}\left(Y_{k, i}^{2}-Y_{c}^{2}\left(\tau_{k, i}, U_{10}, S, \tau_{0}\right)\right)^{2}
$$

in which $Y_{c}^{2}$ is the function from (12) computed with the set of estimated parameters $\left\{U_{10}, S, \tau_{0}\right\}$. The $k$ th waveform was rejected from the ensemble when $r_{k}$ exceeded a threshold. The complete ensemble (less the waveforms which were rejected) was then processed through the least squares estimator a second 


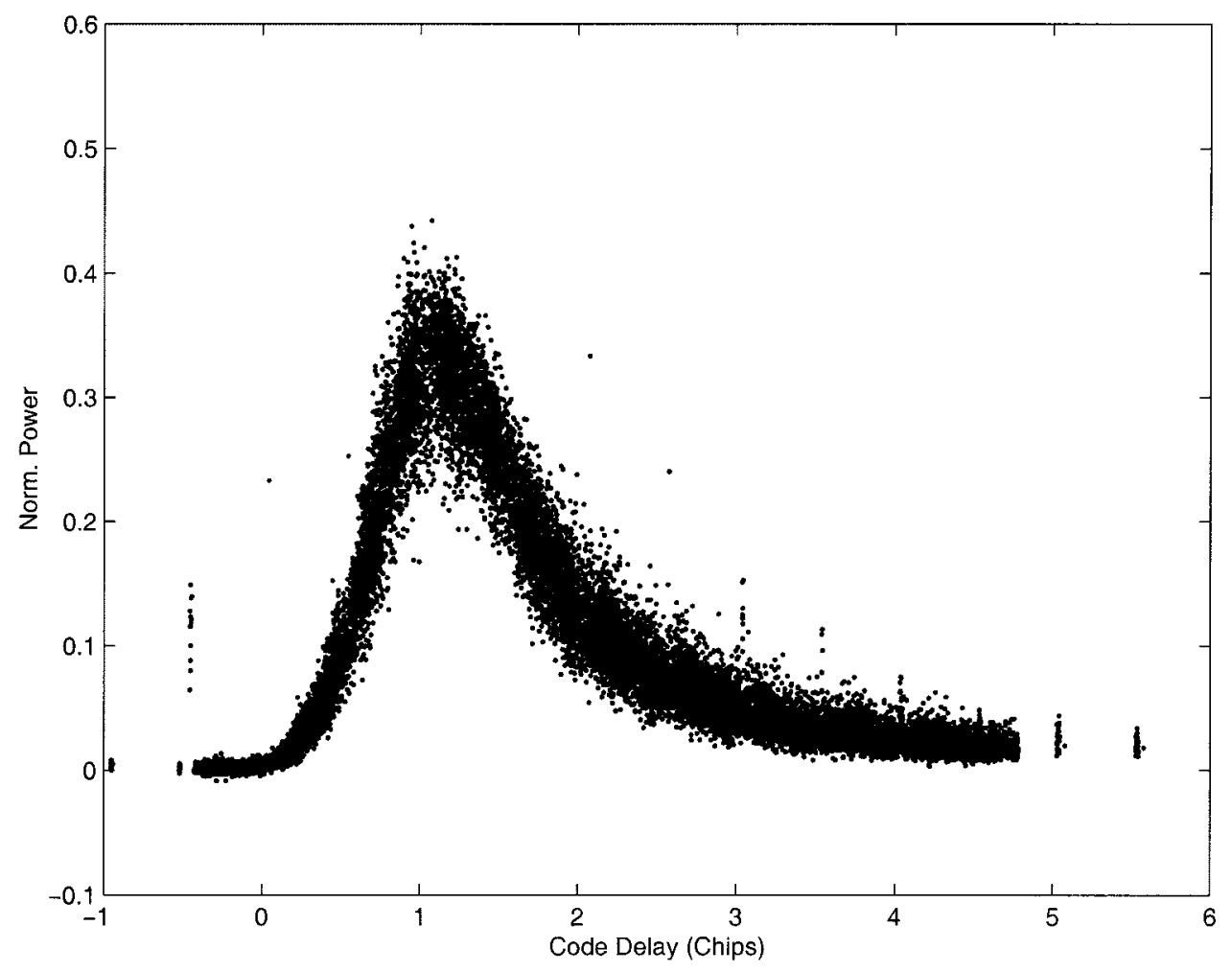

Fig. 12. Corrected waveform, following realignment of centroid.

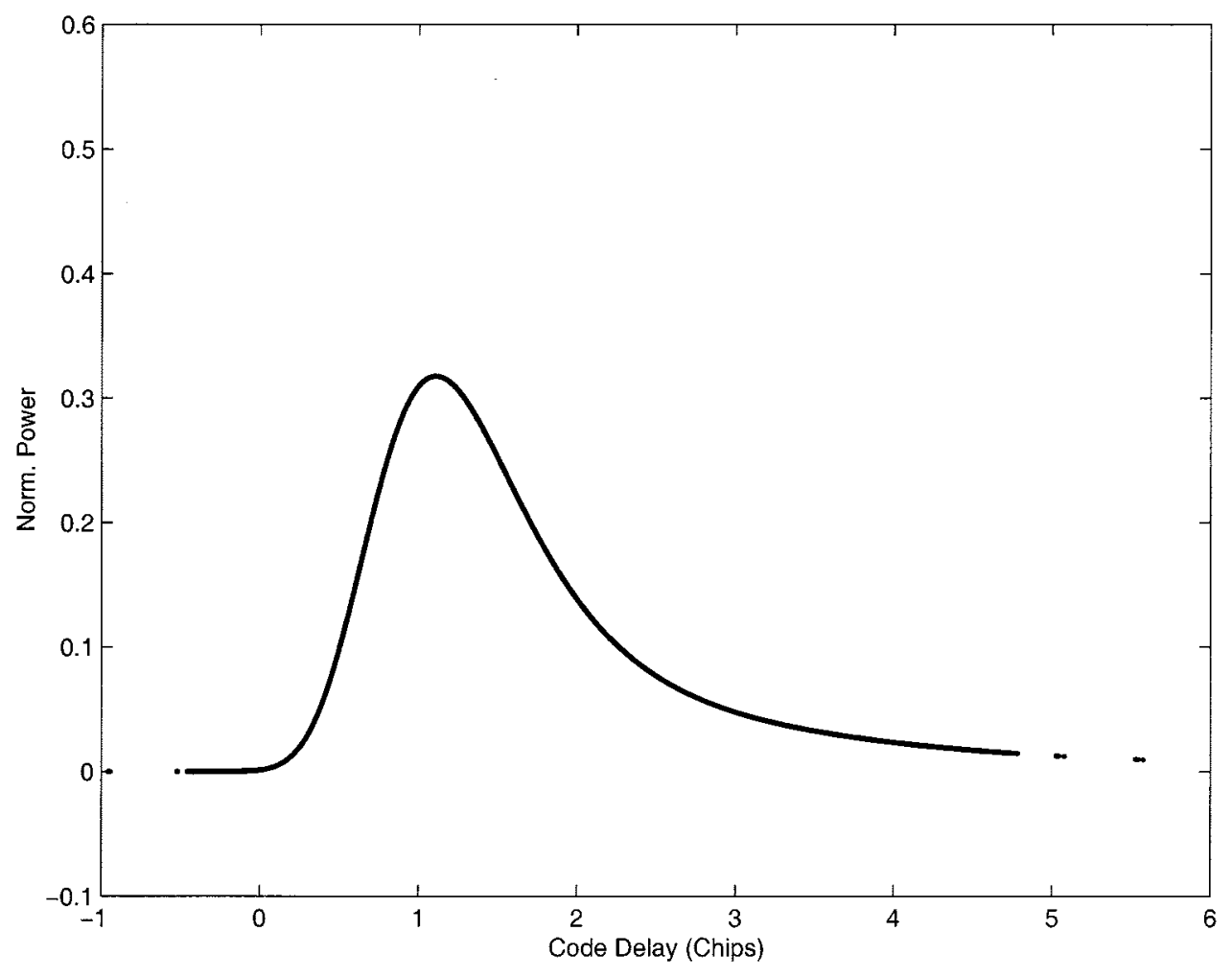

Fig. 13. Least squares fit of (12) to waveform.

time. Fig. 13 shows the resulting best fit waveform to the data from Fig. 12. Fig. 14 shows the post fit residuals for each measured waveform. The threshold value was set by experimentation, and was between approximately equal to between 2.6 and 3.5 times the root mean square of the residuals. All of the sta- tistical interpretations given to these threshold values set for the residuals, or first bin power were based upon the experimentally measured statistics from the PDMR data.

Results from this estimator were compared against the TOPEX wind speed retrievals for the series of TOPEX un- 


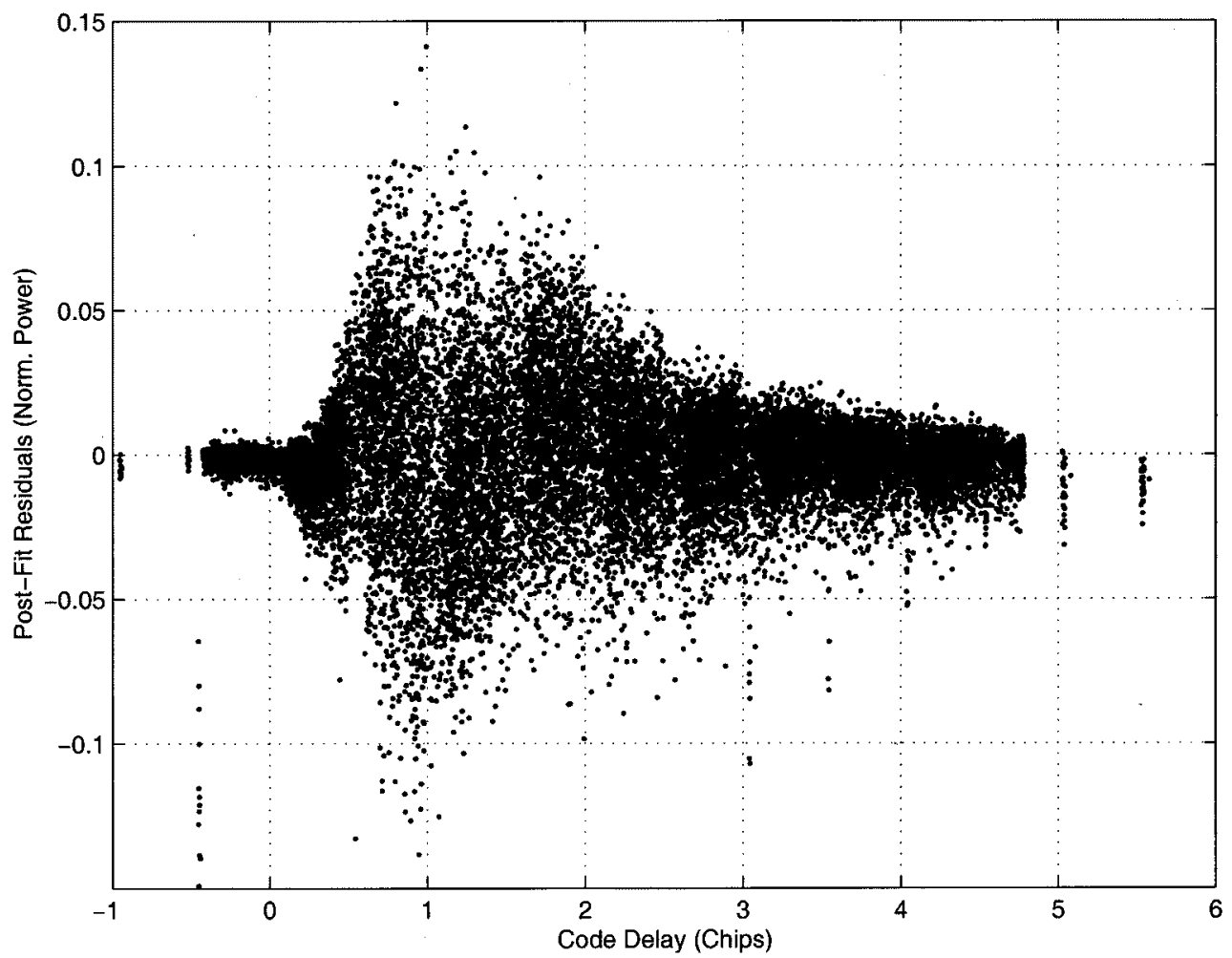

Fig. 14. Residuals from fit of (12) to waveform

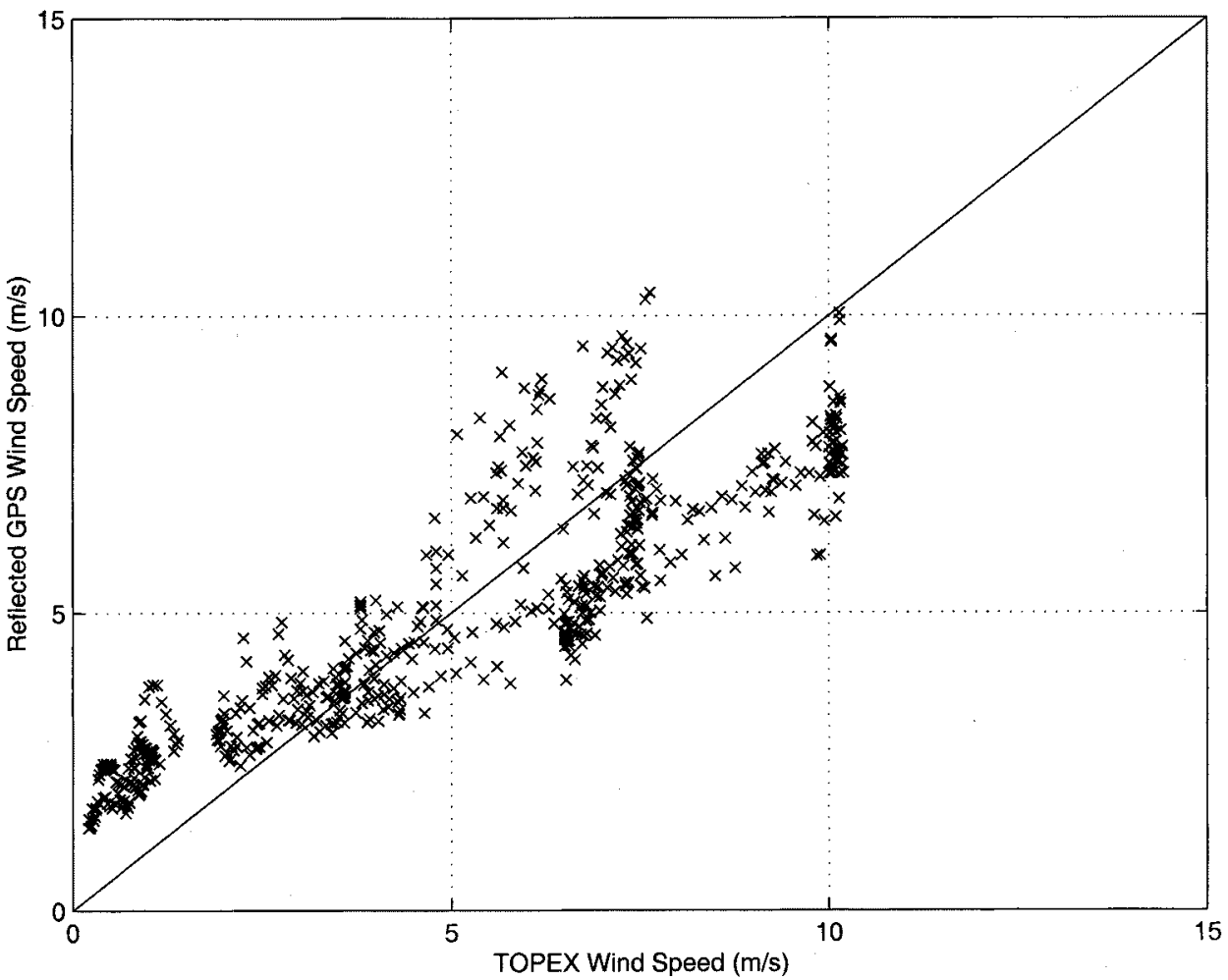

Fig. 15. Comparison of reflected GPS wind speed retrievals versus TOPEX (the solid line, indicating equal wind speeds, is added for comparison).

derflights and against the surface truth measured from buoys during the EOPACE experiment. Fig. 15 shows a comparison between the results of this method operating on PDMR data and TOPEX wind speed retrieval. These retrievals were performed on $10 \mathrm{~s}$ batches of waveform measurements and coefficients $a_{i, j}$ computed for the average altitude and elevation of each GPS satellite from which data were collected.

The longitude of the nearest approach to the TOPEX ground track of the specular point was used to align the two data sets. A simple comparison, assuming the local surface of the Earth to 


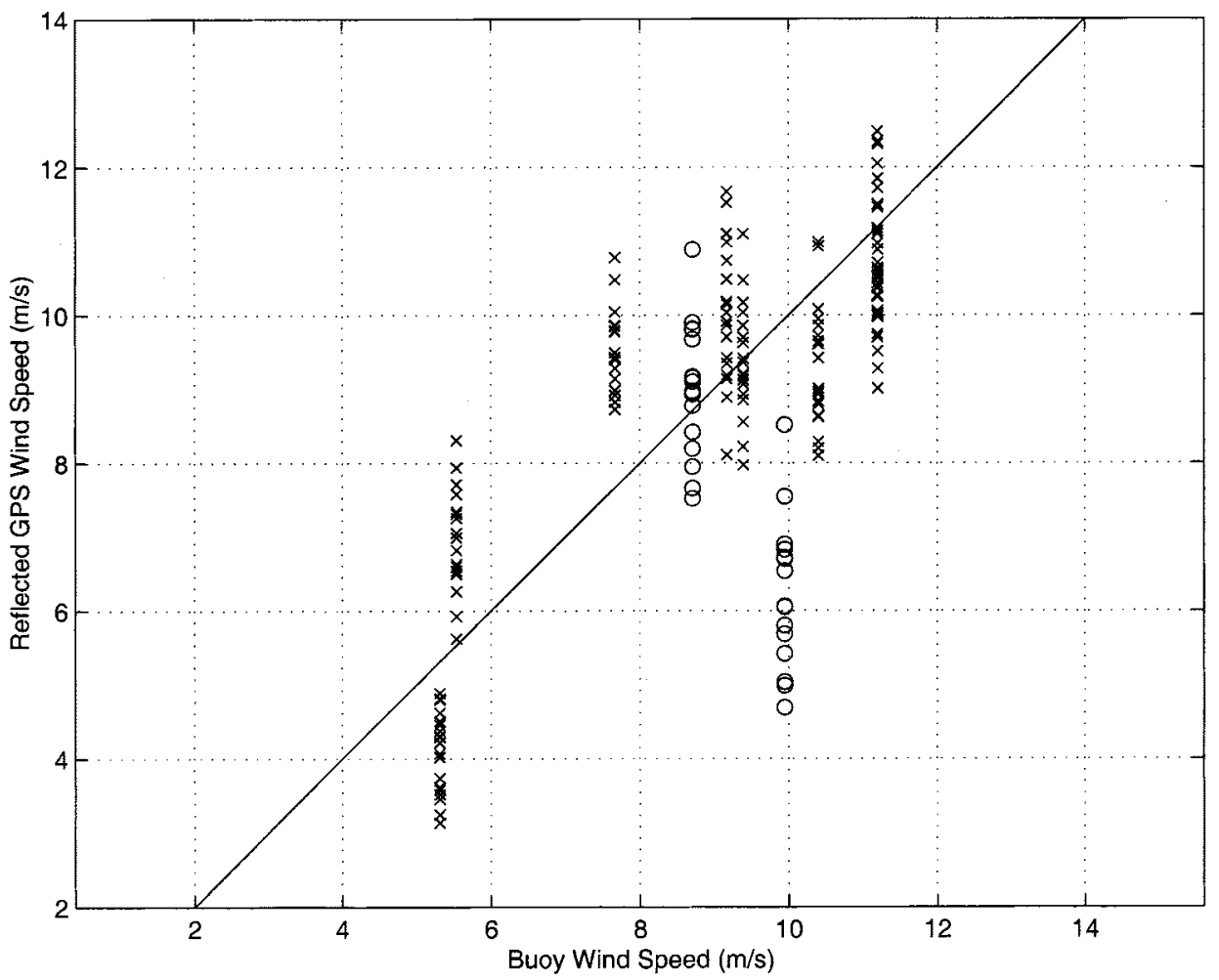

Fig. 16. Comparison of wind speed obtained from reflected GPS measurements with that from recording buoys during the EOPACE experiment in March, 1999 (the solid line, indicating equal wind speeds, is added for comparison).

be flat, was used. The specular point does not lie on exactly the same line as the TOPEX ground track and the approach taken was to compare points which were the closest to each other on the ocean surface. In the near vicinity of each specular point, the TOPEX ground track was approximated as a straight line on a locally flat Earth: $\Delta \phi=m \Delta \lambda+b$, in which $\Delta \phi$ and $\Delta \lambda$ are the differences in latitude and longitude from specular point to points along the ground track. The differences correspond to approximate distances on the ocean surface of $R_{E} \Delta \phi$ and $R_{E} \cos \phi_{S} \Delta \lambda$, respectively. Through a simple minimization procedure, the point on the TOPEX ground track with the closest approach to the specular point at $\left(\phi_{S}, \lambda_{S}\right)$ is

$$
\begin{aligned}
\phi_{N} & =\phi_{S}+m \lambda_{S}+b \\
\lambda_{N} & =\lambda_{S}-\frac{m b}{m^{2}+\cos ^{2} \phi_{S}} .
\end{aligned}
$$

The longitude $\lambda_{N}$ was used to interpolate the TOPEX data and obtain the comparison wind speed for the reflected GPS data retrieval performed on a segment of data with a midpoint at $\left(\phi_{S}, \lambda_{S}\right)$.

Fig. 15 summarizes the comparison of the wind speeds retrieved from matching the reflected GPS waveforms. Segments of data collected at high altitudes $(4 \mathrm{~km}$ or higher) in which the satellite elevation was under $30^{\circ}$ were not used, and segments below $4 \mathrm{~km}$, in which the satellite elevation was under $40^{\circ}$ were also rejected. The justification for the assumption of very weak dependence on wind direction, does not hold true for very low elevation satellites. At the present state, this estimation does not involve wind direction. No data were processed for altitudes below $2.5 \mathrm{~km}$, because the sensitivity to wind speed is not strong at very low altitudes. The solid line on this plot indicates the condition of identical measurements from both methods and was added for comparison. For the ensemble of measurements presented in Fig. 15 the bias in wind speed estimate in comparison to TOPEX was $-0.03 \mathrm{~m} / \mathrm{s}$, and the standard deviation was $1.5 \mathrm{~m} / \mathrm{s}$.

For the EOPACE experiment, segments of data in which the specular point of the reflection was near to a recording buoy were used to evaluate the accuracy of the wind speed retrieval. The Flux buoy was approximately $10 \mathrm{~km}$ from the shore and was expected to give the best comparison with nearby measurements. However, this buoy was not functioning on March 11 and 12 , so the Met buoy (closer to the coast) was used for comparison on those days. The land area surrounding the shoreline was relatively flat, however, and therefore the wind speed measured at all three surface truth locations was found to be similar. Wind direction was monitored at the Duck meteorological station.

Sets of $100 \mathrm{~s}$ of data, approximately centered at the Flux buoy location, were used in these comparisons. Batches of 5 seconds of waveform data from these segments were processed to obtain each parameter estimate. A set of coefficients, $a_{i, j}$ were computed for the series in (12) at the aircraft altitude and the average satellite elevation during the time that the buoy overflight occurred. Only satellites with an elevation greater than 30 deg. were considered.

Results from the EOPACE experiment are summarized in Fig. 16. Again, the solid line was added to indicate the ideal condition of identical measurements from both methods. The bias and standard deviation of the complete set of EOPACE measurements presented in Fig. 16 was $0.09 \mathrm{~m} / \mathrm{s}$ and $1.3 \mathrm{~m} / \mathrm{s}$, re- 
spectively. Note that on March 1, 1999, (the data indicated by "O" on Fig. 16) the wind was from the East $\left(272^{\circ}\right)$. The large error in that set of measurements may therefore be explained by nonfully developed seas.

\section{CONCLUSIONS AND Future WorK}

These experiments have demonstrated reliable retrievals of geophysical data using the shape of the correlation function waveform recorded from forward-scattered GPS signals. The two airborne campaigns using the reflected GPS measurement in conjunction with an existing remote sensing satellite or buoy measurements provided a comparison of the wind speed retrieval techniques with reference measurements. Future experimentation, however, should be conducted under better known conditions and with better surface truth. Retrieval methods could be improved through better electromagnetic models of the reflection process, specifically effects of polarization. Also, the statistics of describing the random rough surface (mean square slope) could be the parameters that are estimated as opposed to making a direct estimate of geophysical data such as wind speeds. This would provide a more fundamental model based on the scattering physics and then geophysical data (wind vectors) could be estimated as a second step. This step may be improved through determining empirical relationships specific to the forward scattered GPS measurement, similar to that done earlier for optical measurements [31]. However, the potential to improve this measurement through empirical corrections should not obscure the fact that good results have already been obtained using a model based upon theory alone.

Another area of improvement is the incorporation of multiple satellites into the same estimator for measurement situations in which the specular point locations are close enough together that they can be assumed to be samples of the same surface process. An understanding of the statistics of the waveform power samples from both experimental and theoretical sources would improve the estimator design and provide a rational basis for selecting weights on the data and thresholds on the residual monitoring. The directional information may also be retrieved from the reflected signal through the mapping of the waveform in both code delay and Doppler frequency as has recently been proposed [32]. This would require enhancements in the receiver architecture and is the next step in the development of this hardware. Use of higher-order statistics may also bring to light additional information in the signal. Finally, the limits of the geometric optics assumption in these scattering models should be investigated.

\section{REFERENCES}

[1] J. L. Garrison, S. J. Katzberg, and M. I. Hill, "Effect of sea roughness on bistatically scattered range coded signals from the global positioning system," Geophys. Res. Lett., vol. 25, no. 13, pp. 2257-2260, July 1, 1998.

[2] M. Martin-Neira, "A passive reflectometry and interferometry system (PARIS): Application to ocean altimetry," ESA J., vol. 17, pp. 331-355, 1993.

[3] — , "Altimetry method," U.S. Patent 5 546 087, Aug. 13, 1996.

[4] V. U. Zavorotny and A. G. Voronovich, "Scattering of GPS signals from the ocean with wind remote sensing application," IEEE Trans. Geosci. Remote Sensing., vol. 38, pp. 951-964, Mar. 2000.
[5] E. D. Kaplan, Ed., Understanding GPS Principles and Applications. Reading, MA: Artech House, 1996.

[6] B. W. Parkinson, J. J. Spilker, P. Axelrad, and P. Enge, Eds., Global Positioning System: Theory and Applications. Washington, DC: AIAA, 1996.

[7] S. J. Katzberg and J. L. Garrison, "Utilizing GPS to determine the ionospheric delay over the ocean," Tech. Rep. NASA TM-4750, Washington, Dec. 1996.

[8] B. Lin, S. J. Katzberg, J. L. Garrison, and B. Wielicki, "The relationship between the GPS signals reflected from sea surfaces and the surface winds: Modeling results and comparisons with aircraft measurements," J. Geophys. Res., vol. 104, no. C9, pp. 20 713-20, 727, Sept. 15, 1999.

[9] C. Cox and W. Munk, "Measurement of the roughness of the sea surface from photographs of the Sun's glitter," J. Opt. Soc. Amer, vol. 44, pp. 838-850, Nov. 1954

[10] GEC Plessey Semiconductors, Global Positioning Products Handbook, August 1996.

[11] J. L. Garrison and S. J. Katzberg, "The application of reflected GPS signals to ocean remote sensing," Remote Sens. Environ., vol. 73, pp. 175-187, 2000.

[12] "Extending the National Geodetic Survey standard orbit formats," Nat. Ocean. Atmos. Admin. (NOAA), Tech. Rep. NOS 133 NGS 46, Washington, DC, Nov. 1989

[13] G. S. Brown, H. R. Stanley, and N. A. Roy, "The wind speed measurement capability of space born radar altimeters," IEEE J. Oceanic Eng., vol. 6, pp. 59-63, 1981.

[14] D. B. Chelton and P. J. McCabe, "A review of satellite measurement of sea surface wind speed with a proposed algorithm," J. Geophys. Res., vol. 90, pp. 4707-4720, 1985.

[15] D. B. Chelton and F. J. Wentz, "Further development of an improved altimeter wind speed algorithm," J. Geophys. Res., vol. 91, pp. 14250-14260, 1986

[16] E. Dobson, F. Monaldo, and J. Goldhirst, "Validation of Geosat altimeter derived wind speeds and significant wave heights using buoy data," $J$. Geophys. Res., vol. 92, pp. 10719-10731, 1987.

[17] M. I. Skolnik, Introduction to Radar Systems. New York: McGrawHill, 1962.

[18] "TOPEX ground system science algorithm specification," Jet Propul. Lab. Pub., JPL 633-708, D-7075, Rev. A, Change 1, Pasadena, CA, 1990.

[19] M. I. Witter and M. I. Chelton, "A Geosat altimeter wind speed algorithm and a method for altimeter wind speed algorithm development," J. Geophys. Res., vol. 96, pp. 8853-8860, 1991.

[20] R. Barnada, User's Handbook (PO.DACC Merged GDR (TOPEX/Poseidon). Pasadena, CA: Jet Propul. Lab., Calif. Inst. Technol. Pub. Ver. 1.0, D11007.

[21] S. F. Clifford, V. I. Tatarskii, A. G. Voronovich, and V. U. Zavorotny, "GPS sounding of ocean surface waves: Theoretical assessment," in Proc. IEEE Int. Geoscience and Remote Sensing Symp.: Sensing and Managing the Environment. Piscataway, NJ: IEEE, 1998, pp. 2005-2007.

[22] V. U. Zavorotny and A. G. Voronovich, "Bistatic radar scattering from an ocean surface in the small-slope approximation," in Proc. IEEE Int. Geoscience and Remote Sensing Symp.: Remote Sensing of the System Earth-A Challenge for the 21st Century. Piscataway, NJ: IEEE, 1999, pp. 2419-2421.

[23] _ "Two-scale model and ocean radar Doppler spectra at moderateand low-grazing angles," IEEE Trans. Antennas Propagat., vol. 46, pp. 84-92, 1998.

[24] T. Elfouhaily, B. Chapron, K. Katsaros, and D. Vandemark, "A unified directional spectrum for long and short wind-driven waves," J. Geophys. Res., vol. 102, pp. 15781-15796.

[25] D. Lemaire, P. Sobieski, and A. Guissard, "Full-range sea surface spectrum in nonfully developed state for scattering calculations," IEEE Trans. Geosci. Remote Sensing, vol. 37, pp. 1038-1051, Mar. 1999.

[26] B. Hofmann-Wellenhof, H. Lichtenegger, and J. Collins, GPS Theory and Practice. New York: Springer-Verlag, 1992.

[27] M. Armatys, A. Komjathy, P. Axelrad, and S. J. Katzberg, “A comparison of GPS and scatterometer sensing of ocean wind speed and direction," in IEEE Int. Geoscience and Remote Sensing Symp., Honolulu, HI, July 24-28, 2000.

[28] MATLAB Optimization Toolbox Users Guide. Natick, MA: The Mathworks, Inc., 1997, pp. 2-20-221.

[29] T. F. Coleman and Y. Li, "On the convergence of reflective Newton methods for large-scale nonlinear minimization subject to bounds," Math. Programm., vol. 67, no. 2, pp. 189-224, 1994.

[30] — " "An interior, trust region approach for nonlinear minimization subject to bounds," SIAM J. Optim., vol. 6, pp. 418-445, 1996. 
[31] J. A. Shaw and J. H. Churnside, "Scanning-laser glint measurements of sea-surface slope statistics," Appl. Opt., vol. 36, no. 18, pp. 4202-4213, June 1997.

[32] M. Armatys, "Delay mapping and frequency selection for estimation of ocean surface parameters," in Proc. GPS Applications to the Structure and Dynamics of the Earth's Oceans and Ionosphere, Arcadia, CA, Dec. $1-3,1998$.

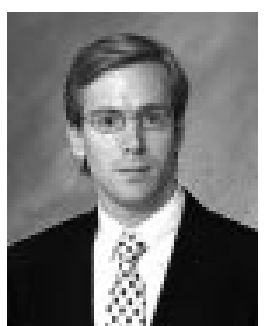

James L. Garrison (M'98) received the B.S. degree from Rensselaer Polytechnic Institute, Rensselaer, NY, in 1988, the M.S. degree from Stanford University, Stanford, CA, in 1990, and the Ph.D. degree in aerospace engineering sciences from the University of Colorado, Boulder, in 1997.

In 2000, he joined the Faculty of the School of Aeronautics and Astronautics, Purdue University, West Lafayette, IN, as an Assistant Professor. From 1998 to 2000, he was in the Applied Engineering and Technology Directorate, NASA's Goddard Space Flight Center, Greenbelt, MD. From 1988 until 1998, he was employed by NASA's Langley Research Center, Hampton, VA. His research focuses on new applications of GPS in the areas of remote sensing and orbit navigation.

Dr. Garrison is a member of the IEEE Geoscience and Remote Sensing Society, the Institute of Navigation (ION), and the American Geophysical Union (AGU). He is also a Senior Member of the American Institute of Aeronautics and Astronautics, and shares, with Dr. S. J. Katzberg, a NASA Exceptional Space Act Award for his work on bistatic remote sensing utilizing GPS signals.

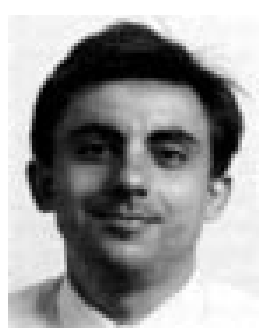

Attila Komjathy received the Ph.D. degree from the Department of Geodesy and Geomatics Engineering, University of New Brunswick, Fredericton, NB, Canada, in 1997.

He is currently a Member of Technical Staff, NASA Jet Propulsion Laboratory (JPL), Pasadena, $\mathrm{CA}$, specializing in atmospheric and ionospheric remote sensing techniques using the Global Positioning System. Prior to joining JPL in July 2001, he worked on the utilization of GPS reflected signals as a Research Associate at the University of Colorado's Center for Astrodynamics Research for four years.

Dr. Komjathy's dissertation on the ionospheric total electron content mapping using GPS won the 1998 Canadian Governor General's Gold Medal.
Valery U. Zavorotny received the degree in radio physics from Gorky State University, Gorky, Russia, in 1971, and the Ph.D. degree in physics and mathematics from the Institute of Atmospheric Physics, USSR Academy of Sciences, Moscow, Russia.

From 1971 to 1990, he was a Research Scientist with the Institute of Atmospheric Physics, USSR Academy of Sciences. In 1990, he joined Lebedev Physical Institute, Moscow. Since 1991, he has been a Research Scientist with the Cooperative Institute for Research in Environmental Sciences and the Environmental Technology Laboratory, University of Colorado, National Oceanic and Atmospheric Administration (NOAA), Boulder, CO. His research interests are in wave scattering from rough surfaces, optical and radio wave propagation through random media, and ocean and atmospheric remote sensing techniques.

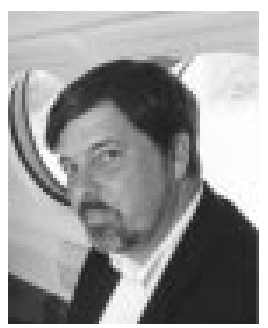

Stephen J. Katzberg received the B.S.E.E. degree from the Massachusetts Institute of Technology, Cambridge, in 1965, and the M.S.E.E. and Ph.D degrees in electrical engineering from the University of Virginia, Charlottesville, in 1967 and 1970, respectively.

$\mathrm{He}$ is currently an Electrical Engineer with the NASA Langley Research Center, Hampton, VA. His experience is in the theory and analysis of advanced techniques for remote sensing including: modeling of electro-optical systems such as electromechanica scanners (Viking Lander Camera, TBAMS), laser-based systems (Laser Hetrodyne Spectrometer), interferometers (DASI, ITS), and modeling of the interaction between radiation fields and surfaces (GPS surface reflections, ESTAR).

Dr. Katzberg was awarded a NASA Exceptional Space Act Award (along with Dr. J. L. Garrison) for his research in GPS surface reflections. He also holds a NASA Exceptional Achievement Medal and an AIAA Distinguished Leadership Award. 